\title{
THE SMALLEST REPRESENTATIONS OF NON-LINEAR COVERS OF ODD ORTHOGONAL GROUPS
}

\author{
HUNG YEAN LOKE AND GORDAN SAVIN
}

\begin{abstract}
We construct the smallest genuine representations of a non-linear covers of the group $\mathrm{SO}^{0}(p, q)$ where $p+q$ is odd. We determine correspondences of infinitesimal characters arising from restricting the smallest representations to dual pairs $\mathfrak{s o}(p, a) \oplus$ $\mathfrak{s o}(b)$ where $a+b=q$.
\end{abstract}

\section{INTRODUCTION}

Let $p$ and $q$ be two positive integers $\geq 2$, and let $G_{p, q}$, or simply $G$, be the central extension of $\mathrm{SO}^{0}(p, q)$ such that the maximal compact subgroup is $K=\operatorname{Spin}(p) \times \operatorname{Spin}(q)$. This extension is the universal central extension if $p, q \neq 2$. Assume now that $p+q$ is odd. In particular, $p$ and $q$ have different parity. Without any loss of generality we shall assume that $p$ is odd and $q$ even. Let $Z_{G}$ be the center of $G$. Then

$$
Z_{G} \cong \mathbb{Z} / 2 \mathbb{Z} \times \mathbb{Z} / 2 \mathbb{Z}
$$

However, there exists a unique element of order 2 in $Z_{G}$, denoted simply by -1 , such that $G /\langle-1\rangle$ is a linear group. An irreducible representation $\pi$ of $G$ is called genuine if $-1 \in Z_{G}$ acts as multiplication by -1 on $\pi$. The first part of this paper is devoted to constructing and establishing properties of one genuine representation $V$ of the group $G$ if $p-1<q$, two genuine representations $V^{+}$and $V^{-}$of $G$ if $p-1>q$, and four genuine representations $V^{+}, V^{-}, V_{o}^{+}$and $V_{o}^{-}$if $p-1=q$. If $p-1=q$ then the group $G$ is split with the absolute root system $\mathrm{B}_{q}$. In the special case when $q=2$ and $p=3$ then $G$ is isomorphic to the metaplectic group $\widetilde{\mathrm{Sp}_{4}}(\mathbb{R})$, and the four representations are irreducible components of two oscillator representations. Thus, our representations can be viewed as a natural generalization of the oscillator representation to odd orthogonal groups.

The first step in the construction is an explicit description of $K$-types of these representations. To this end, we need to recall a description of irreducible representations of $\operatorname{Spin}(n)$. Let $\Lambda(n)$ be the set of all highest weight of finite dimensional representations. We realize $\Lambda(n)$ as in Bourbaki [Bo], see Section 2 of this paper. In particular, any highest weight $\mu$ is given by

$$
\mu=\left(x_{1}, \ldots, x_{\left[\frac{n}{2}\right]}\right)
$$

where $x_{i}$ are either all integers or half-integers. Let $-1_{n}$ be the unique element in $\operatorname{Spin}(n)$ such that $\operatorname{Spin}(n) /\left\langle-1_{n}\right\rangle \cong \mathrm{SO}(n)$. We can divide all irreducible representations of

1991 Mathematics Subject Classification. 22E46, 22E47. 
$\operatorname{Spin}(n)$ into two classes, depending whether $-1_{n}$ acts as 1 or -1 . This corresponds to writing

$$
\Lambda(n)=\Lambda(n, 0) \cup \Lambda\left(n, \frac{1}{2}\right)
$$

where $\Lambda(n, 0)$ consists of integral and $\Lambda\left(n, \frac{1}{2}\right)$ of half-integral highest weights. Let $\tau_{n}^{\mu}$ denote the irreducible representation of $\operatorname{Spin}(n)$ with the highest weight $\mu$. Let $\mathcal{Z}_{n}$ be the center of the enveloping algebra of $\operatorname{Spin}(n)$. Recall that the infinitesimal character $\chi$ defines a ring homomorphism $\chi: \mathcal{Z}_{n} \rightarrow \mathbb{C}$.

We are now ready to describe the $K$-types of our representations. Assume that $p-1<q$. In Section 2, we define a surjective homomorphism

$$
j: \mathcal{Z}_{q} \rightarrow \mathcal{Z}_{p}
$$

Let $\chi$ be the infinitesimal character of an irreducible representation $\tau$ of $\operatorname{Spin}(p)$. Then the composition $\chi \circ j$ is an infinitesimal character of $\operatorname{Spin}(q)$, but not necessarily corresponding to a finite dimensional representation of $\operatorname{Spin}(q)$. More precisely, for every $\lambda$ in $\Lambda(p, 0)$ define

$$
\left\{\begin{array}{l}
A(\lambda)=\lambda+\frac{(q-p)}{2}(1, \ldots, 1) \in \Lambda\left(p, \frac{1}{2}\right) \\
B(\lambda)=\left(\lambda_{1}, \ldots, \lambda_{\frac{p-1}{2}}, 0, \ldots, 0\right) \in \Lambda(q, 0) .
\end{array}\right.
$$

Then $\chi \circ j$ is an infinitesimal character of a finite dimensional representation of $\operatorname{Spin}(q)$ if and only if the highest weight of the irreducible representation $\tau$ of $\operatorname{Spin}(p)$ is $A(\lambda)$ for some $\lambda$ in $\Lambda(p, 0)$. If that is the case, then $\chi \circ j$ is the infinitesimal character of the irreducible representation of $\operatorname{Spin}(q)$ with the highest weight $B(\lambda)$. We now set $V$ - a potential $(\mathfrak{g}, K)$-module - to be the $K$-module

$$
V=\bigoplus_{\lambda \in \Lambda(p, 0)} \tau_{p}^{A(\lambda)} \otimes \tau_{q}^{B(\lambda)}
$$

Since $-1 \in Z_{G}$ is given by $\left(-1_{p},-1_{q}\right) \in K$ we see that $V$ must correspond to a genuine representation of $G$, once we have defined an action of $\mathfrak{s o}(p, q)$, the Lie algebra of $G$, on $V$.

If $p-1 \geq q$ then the map $j$ goes in the opposite direction. The main difference here lies in the fact that $j$ is not surjective anymore. In particular, two different infinitesimal characters of $\operatorname{Spin}(q)$ pull back to the same infinitesimal character of $\operatorname{Spin}(p)$. As a consequence, we can construct two potential $(\mathfrak{g}, K)$-modules denoted by $V^{+}$and $V^{-}$. The story has an additional twist if $p-1=q$ enabling us to write down four potential modules in all, here. We refer the reader to Section 2 for details.

The structure of $K$-types is similar to the structure of $K$-types of representations of $\mathrm{SO}^{0}(p, q)$, where $p+q$ is even, that are local theta lifts of one dimensional unitary characters of $\widetilde{\operatorname{Sp}}_{2 n}(\mathbb{R})$ (see $[\mathrm{KO}]$, [HL] and $[\mathrm{ZH}]$ ).

From the explicit description of $K$-types it is not too difficult to determine the associated variety of $V$. Indeed, consider the nilpotent orbit of $\mathrm{SO}_{p+q}(\mathbb{C})$ corresponding to the partition $\left(2^{p-1}, 1^{q-p+2}\right)$. It has a (unique) real form $\mathcal{O}_{2^{p-1}}$ for the group $\operatorname{SO}^{0}(p, q)$. Let 


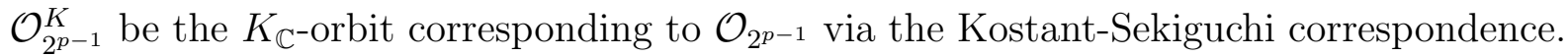
We have the following:

Theorem 1.1. Recall that $K=\operatorname{Spin}(p) \times \operatorname{Spin}(q)$ with $p$ odd and $q$ even. Suppose $p-1<q$. The $K$-module $V$ extends to an irreducible and unitarizable $(\mathfrak{s o}(p, q), K)$ module. Moreover:

(i) The infinitesimal character of $V$ is

$$
\mu_{p, q}=\left(\frac{p-1}{2}, \frac{p-3}{2}, \ldots, 1, \frac{q-1}{2}, \frac{q-3}{2}, \ldots, \frac{1}{2}\right) .
$$

(ii) The associated variety of $V$ is the closure of $\mathcal{O}_{2^{p-1}}^{K}$.

(iii) The annihilator of $V$ in the enveloping algebra is the unique maximal ideal $J_{\max }$ with the infinitesimal central character $\mu_{p, q}$.

(iv) The module $V$ is the unique $(\mathfrak{s o}(p, q), K)$-module with the $K$-types as in (1).

Here we remark that $\mathfrak{s o}(p, q)$ is the real Lie algebra of $G_{p, q}$. The complexification of $\mathfrak{s o}(p, q)$ will be denoted by $\mathfrak{s o}_{p+q}(\mathbb{C})$.

It follows, from a result of Schimd and Vilonen [SV], that the wave front set of $V$ is the real orbit $\mathcal{O}_{2^{p-1}}$. This, combined with a result of Huang and $\mathrm{Li}[\mathrm{HL}]$, justifies the use of the attribute smallest in the title of our paper.

Analogous results hold for $V^{+}, V^{-}$and $V_{o}^{+}, V_{o}^{-}$. It must be noted, however, that our results overlap with some already existing in the literature. For example, if $p=3$ then $V$ is the minimal representation of $G_{3, q}$ constructed in [Sa], [To] and [BKo]. If $p-1 \geq q$ then representations $V^{+}$and $V^{-}$were constructed by Knapp [Kn] and further studied by Trapa [T] by methods of cohomological induction. Our method is based on a simple observation that $V$ is admissible for $\operatorname{Spin}(p)$. Such phenomenon is called discretely decomposable restriction in [Ko2]. In particular, the restriction of $V$ to $\mathfrak{s o}(p, 1)$ decomposes as a direct sum of irreducible representations. We exploit this observation to define an explicit action of $\mathfrak{s o}(p, 1)$ on $V$. This then defines an action of $\mathfrak{s o}(p, q)$ on $V$ because $\mathfrak{s o}(p, q)$ is generated by $\mathfrak{s o}(p, 1)$ and $\mathfrak{s o}(q)$.

We then extend $V$ to a $(\mathfrak{s o}(p, q), \operatorname{Spin}(p) \times \mathrm{O}(q))$-module. This extension is needed for the second part of this paper which is devoted to dual pair correspondences arising from restricting $V$ to dual pairs

$$
\mathfrak{s o}(p, a) \times \mathrm{O}(b), \quad a+b=q .
$$

Using our explicit description of $V$ we can show that the Theta-lift of any finite dimensional irreducible representation of $\mathrm{O}(b)$ is irreducible. See Theorem 9.1 and Remark 9.2. We build on this to establish a correspondence of infinitesimal characters. Of course, if $a=0$ and $b=p$ then the correspondence of infinitesimal characters is given by $j$. In order to describe a general result let

$$
\rho_{n}=\frac{1}{2}(n-2, n-4, n-6, \ldots) \in \Lambda(n)
$$


denote the half sum of positive roots of $\mathfrak{s o}(n)$. Given $\beta=\left(\beta_{1}, \ldots, \beta_{r}\right)$ and $\gamma=\left(\gamma_{1}, \ldots, \gamma_{s}\right)$, we will denote $\left(\beta_{1}, \ldots, \beta_{r}, \gamma_{1}, \ldots, \gamma_{s}\right)$ by $(\beta, \gamma)$ if there is no fear of confusion. Let

$$
\delta_{p, q-1}=\left(\rho_{p}, \rho_{q}\right) .
$$

We remark here that $\delta_{p, q-1}$ is the infinitesimal character of two (smallest) genuine representations of $G_{p, q-1}$ obtained as Theta-lifts of the trivial and the sign representations of $\mathrm{O}(1)$. (Compare this with (7.2.1) in [KO] for the ladder representations of the even orthogonal groups.) This statement is in essence a special case $(b=1)$ of the following theorem.

Theorem 1.2. Assume that $a+b=q$. The representation $V$ establishes the following correspondence of infinitesimal characters for the dual pair $\mathfrak{s o}(p, a) \times \mathfrak{s o}(b)$ :

$$
\left\{\begin{array}{l}
\left(\lambda_{1}, \ldots, \lambda_{\frac{p-1}{2}}, \rho_{a-1}\right) \longleftrightarrow\left(\lambda_{1}, \ldots, \lambda_{\frac{p-1}{2}}, \rho_{b-p+1}\right) \text { if } b \geq p . \\
\left(\lambda_{1}, \ldots, \lambda_{\frac{b}{2}}, \mu_{p-b, q-b}\right) \longleftrightarrow\left(\lambda_{1}, \ldots, \lambda_{\frac{b}{2}}\right) \text { if } b<p \text { and } b \text { is even. } \\
\left(\lambda_{1}, \ldots, \lambda_{\frac{b-1}{2}}, \delta_{p-b+1, q-b}\right) \longleftrightarrow\left(\lambda_{1}, \ldots, \lambda_{\frac{b-1}{2}}\right) \text { if } b<p \text { and } b \text { is odd } .
\end{array}\right.
$$

Warning: We are not claiming here that the correspondence of infinitesimal characters is one to one. For example, if $b$ is even and $b<p$ then the infinitesimal characters $\left(\lambda_{1}, \ldots, \lambda_{\frac{b}{2}}\right)$ and $\left(\lambda_{1}, \ldots,-\lambda_{\frac{b}{2}}\right)$ of $\mathfrak{s o}(b)$ correspond to the same infinitesimal character of $\mathfrak{s o}(p, a)$.

This paper is motivated by two beautiful papers of Bump, Friedberg and Ginzburg [BFG1] and [BFG2] where, for split groups, a $p$-adic version of $V$ is constructed. In particular, the construction of $K$-types using the correspondence of infinitesimal characters induced by $j$ is a real analogue of the correspondence of Satake parameters obtained in [BFG2].

Acknowledgment. We would like to thank Peter Trapa for some very insightful discussions and the referee for careful reading and comments. The first author would like to thank the hospitality of the mathematics department at University of Utah while part of this paper was written. He is supported by an NUS grant R-146-000-085-112. The second author is supported by an NSF grant DMS-0551846.

\section{Potential $(\mathfrak{g}, K)$-modules}

We use the standard realization of root systems of classical groups as in Bourbaki [Bo].

Representations of $\operatorname{Spin}(p)$ where $p$ is odd. Let $-1_{p}$ be the unique element in $\operatorname{Spin}(p)$ such that $\operatorname{Spin}(p) /\left\langle-1_{p}\right\rangle \cong \mathrm{SO}(p)$. Recall that the highest weight of an irreducible finitedimensional representation of $\operatorname{Spin}(p)$ is given by

$$
\lambda=\left(x_{1}, \ldots, x_{\frac{p-1}{2}}\right)
$$


where $x_{i} \in \frac{1}{2} \mathbb{Z}, x_{1} \geq \ldots \geq x_{\frac{p-1}{2}} \geq 0$ and $x_{1} \equiv \ldots \equiv x_{\frac{p-1}{2}}(\bmod \mathbb{Z})$. The corresponding irreducible representation is denoted by $\tau_{p}^{\lambda}$. Let $\Lambda(p)$ be the set of all highest weights. We can write

$$
\Lambda(p)=\Lambda(p, 0) \cup \Lambda\left(p, \frac{1}{2}\right)
$$

where if $\lambda \in \Lambda(p, e)$ then $\lambda_{i} \in e+\mathbb{Z}$. Note that $-1_{p}$ acts as 1 on $\tau_{p}^{\lambda}$ if and only if $\lambda$ is in $\Lambda(p, 0)$. The center $\mathcal{Z}_{p}$ of the enveloping algebra is equal to

$$
\mathcal{Z}_{p} \cong \mathbb{C}\left[\omega_{1}, \omega_{2}, \ldots, \omega_{\frac{p-1}{2}}\right]
$$

where $\omega_{k}$ is the $k$-th symmetric function in $x_{1}^{2}, \ldots, x_{\frac{p-1}{2}}^{2}$. The value of an element $\omega$ in $\mathcal{Z}_{p}$ on the irreducible representation $\tau_{p}^{\lambda}$ is given by evaluating the polynomial $\omega$ on $\lambda+\rho_{p}$ where where $\rho_{p}$ is the half sum of positive roots as defined in (2).

Representations of $\operatorname{Spin}(q)$ where $q$ is even. Let $-1_{q}$ be the unique element in $\operatorname{Spin}(q)$ such that $\operatorname{Spin}(q) /\left\langle-1_{q}\right\rangle \cong \mathrm{SO}(q)$. Recall that the highest weight of an irreducible finitedimensional representation of $\operatorname{Spin}(q)$ is given by

$$
\lambda=\left(x_{1}, \ldots, x_{\frac{q}{2}}\right)
$$

where $x_{i} \in \frac{1}{2} \mathbb{Z}, x_{1} \geq \ldots \geq x_{\frac{q-2}{2}} \geq\left|x_{\frac{q}{2}}\right|$ and $x_{1} \equiv \ldots \equiv x_{\frac{q}{2}}(\bmod \mathbb{Z})$. The corresponding irreducible representation is denoted by $\tau_{q}^{\lambda}$. Let $\Lambda(q)$ be the set of all highest weights. We can write

$$
\Lambda(q)=\Lambda(q, 0) \cup \Lambda\left(q, \frac{1}{2}\right)
$$

where if $\lambda \in \Lambda(q, e)$ then $\lambda_{i} \in e+\mathbb{Z}$. Note that $-1_{q}$ acts as 1 on $\tau_{q}^{\lambda}$ if and only if $\lambda$ is in $\Lambda(q, 0)$.

The center $\mathcal{Z}_{q}$ of the enveloping algebra is equal to

$$
\mathcal{Z}_{q} \cong \mathbb{C}\left[\omega_{1}, \ldots, \omega_{\frac{q-2}{2}}, v_{\frac{q}{2}}\right]
$$

where $\omega_{k}$ is the $k$-th symmetric function in $x_{1}^{2}, \ldots, x_{\frac{q}{2}}^{2}$, and $v_{\frac{q}{2}}=x_{1} \cdot \ldots \cdot x_{\frac{q}{2}}$. The value of the element $\omega$ in $\mathcal{Z}_{q}$ on the irreducible representation $\tau_{q}^{\lambda}$ of $\mathfrak{s o}(q)$ with the highest weight $\lambda$ is equal to $\omega\left(\lambda+\rho_{q}\right)$ where $\rho_{q}$ is the half sum of positive roots as defined in (2).

We are now ready to define $K$-modules in the three cases, as follows:

Case 1: $p-1<q$. We have a surjective map $j: \mathcal{Z}_{q} \rightarrow \mathcal{Z}_{p}$ given by $j\left(\omega_{k}\right)=\omega_{k}$ for $k=1, \ldots, \frac{p-1}{2}$ and $j=0$ on remaining generators of $\mathcal{Z}_{q}$. For every $\lambda=\left(\lambda_{1}, \ldots, \lambda_{\frac{p-1}{2}}\right)$ in $\Lambda(p, 0)$, we define

$$
\left\{\begin{array}{l}
A(\lambda)=\lambda+\frac{q-p}{2}(1, \ldots, 1) \in \Lambda\left(p, \frac{1}{2}\right) \text { and } \\
B(\lambda)=\left(\lambda_{1}, \ldots, \lambda_{\frac{p-1}{2}}, 0, \ldots, 0\right) \in \Lambda(q, 0)
\end{array}\right.
$$


where there are $\frac{q-p+1}{2}$ copies of 0 in $B(\lambda)$. Notice that the infinitesimal characters of $\tau_{p}^{A(\lambda)}$ and $\tau_{q}^{B(\lambda)}$ are matched by $j$, that is, for every $\omega$ in $\mathcal{Z}_{q}$

$$
j(\omega)\left(A(\lambda)+\rho_{p}\right)=\omega\left(B(\lambda)+\rho_{q}\right) .
$$

Moreover, using the explicit description of $j$, one easily checks that there are no other pairs of representations with matching infinitesimal characters. Thus it is natural to consider

$$
V=\bigoplus_{\lambda \in \Lambda(p, 0)} \tau_{p}^{A(\lambda)} \otimes \tau_{q}^{B(\lambda)}
$$

which is a representation of the compact Lie group $\operatorname{Spin}(p) \times \mathrm{SO}(q)$. In this way we have constructed $K$-types of $V$.

Case $2: p-1 \geq q$. We have a surjective map $j: \mathcal{Z}_{p} \rightarrow \mathcal{Z}_{q}$ given by $j\left(\omega_{k}\right)=\omega_{k}$ for $k=1, \ldots, \frac{q-2}{2}, j\left(\omega_{\frac{q}{2}}\right)=v_{\frac{q}{2}}^{2}$ and $j=0$ on remaining generators of $\mathcal{Z}_{q}$, if any. Note that $j$ is not surjective here. Indeed, consider the involution $\sigma$ of $\Lambda(q)$ (and of $\mathcal{Z}_{q}$ ) defined by defined by

$$
\sigma\left(x_{1}, \ldots, x_{\frac{q-2}{2}}, x_{\frac{q}{2}}\right)=\left(x_{1}, \ldots, x_{\frac{q-2}{2}},-x_{\frac{q}{2}}\right) .
$$

Then the image of $\mathcal{Z}_{p}$ is equal to the subalgebra of $\sigma$-invariant polynomials in $\mathcal{Z}_{q}$. In particular, two representations of $\operatorname{Spin}(q)$ will be matched with one representation of $\operatorname{Spin}(q)$. More precisely, for every $\lambda$ in $\Lambda(q+1,0)$ define

$$
\left\{\begin{array}{l}
A(\lambda)=(\lambda, 0, \ldots, 0) \in \Lambda(p, 0) \\
B^{+}(\lambda)=\lambda+\frac{p-q}{2}(1, \ldots, 1) \in \Lambda\left(q, \frac{1}{2}\right) \\
B^{-}(\lambda)=\sigma\left(B^{+}(\lambda)\right) \in \Lambda\left(q, \frac{1}{2}\right) .
\end{array}\right.
$$

Then the infinitesimal characters of $\tau_{q}^{B^{+}(\lambda)}$ and $\tau_{q}^{B^{-(\lambda)}}$ are matched with the infinitesimal character of $\tau_{p}^{A(\lambda)}$. Moreover, if $p-1>q$, there are no other matching pairs of representations of $\operatorname{Spin}(p)$ and $\operatorname{Spin}(q)$. We can now define $V^{+}$and $V^{-}$, two representations of the compact Lie group $\mathrm{SO}(p) \times \operatorname{Spin}(q)$, by

$$
V^{ \pm}=\bigoplus_{\lambda \in \Lambda(q+1,0)} \tau_{p}^{A(\lambda)} \otimes \tau_{q}^{B^{ \pm}(\lambda)}
$$

The separation of $K$-types into $V^{+}$and $V^{-}$is natural since $V^{+} \otimes \mathfrak{p}$ and $V^{-}$contain no $K$-type in common if $p-1>q$. (Here $\mathfrak{p}=\mathfrak{p}_{0} \otimes \mathbb{C}$ and $\mathfrak{s o}(p, q)=\mathfrak{k}_{0} \oplus \mathfrak{p}_{0}$ is the Cartan decomposition.)

Case 3: $p-1=q$. Finally, if $p-1=q$, there are additional two families of matching pairs

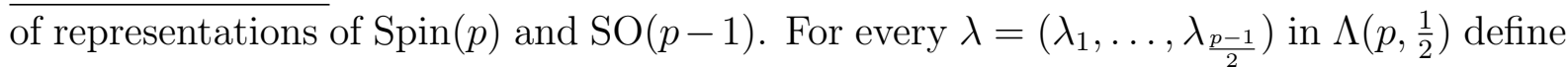

$$
\left\{\begin{array}{l}
B^{+}(\lambda)=\lambda+\frac{1}{2}(1, \ldots, 1) \in \Lambda(p-1,0) \\
B^{-}(\lambda)=\sigma\left(B^{+}(\lambda)\right) \in \Lambda(p-1,0)
\end{array}\right.
$$


Then

$$
V_{o}^{ \pm}=\bigoplus_{\lambda \in \Lambda\left(p, \frac{1}{2}\right)} \tau_{p}^{\lambda} \otimes \tau_{p-1}^{B^{ \pm}(\lambda)}
$$

\section{Representations of $\operatorname{Spin}(n, 1)$}

In this section we review some facts about representations of the group $\operatorname{Spin}(n, 1)$. The maximal compact subgroup is $\operatorname{Spin}(n)$. We identify the Lie algebra $\mathfrak{s o}(n)$ of $\operatorname{Spin}(n)$ with the set of real skew-symmetric $n$ by $n$ matrices. Let $E_{k l}$ be the elementary $n$ by $n$ matrix which is 1 at the $k l$-th entry and zero elsewhere. Let

$$
I_{k l}=E_{l k}-E_{k l} \text {. }
$$

We need to fix some additional notation. As in the previous section, let $\Lambda(n, e)$, where $e=0, \frac{1}{2}$, denote the set of highest weights $\lambda=\left(\lambda_{1}, \ldots, \lambda_{[n / 2]}\right)$ of $\mathfrak{s o}(n)$ where $\lambda_{i} \in \mathbb{Z}+e$. Hence the set of highest weights is $\Lambda(n)=\Lambda(n, 0) \cup \Lambda\left(n, \frac{1}{2}\right)$. If $n$ is even, then we define $\sigma(\lambda)=\left(\lambda_{1}, \ldots, \lambda_{n / 2-1},-\lambda_{n / 2}\right)$. Let

$$
\mathbf{1}_{[n / 2]}=(1, \ldots, 1) \text { and } \mathbf{0}_{[n / 2]}=(0, \ldots, 0)
$$

where there are $\left[\frac{n}{2}\right]$ copies of 1 's and 0 's respectively. We set $\varepsilon_{i}=(0, \ldots, 0,1,0, \ldots, 0)$ where 1 appears at the $i$-th position.

Gelfand-Zetlin basis. We now define the Gelfand-Zetlin basis of a finite dimensional representation of $\mathfrak{s o}(n)$. Our main references are [VK] and [Zhe].

Given $\lambda=\left(\lambda_{1}, \ldots, \lambda_{[n / 2]}\right) \in \Lambda(n, e)$, let $\tau_{n}^{\lambda}$ denote the irreducible representation of $\mathfrak{s o}(n)$ with highest weight $\lambda$ as in the introduction. We will equipped it with an $\mathfrak{s o}(n)$ invariant Hermitian inner product. It is well known that the restriction of $\tau_{n}^{\lambda}$ to $\mathfrak{s o}(n-1)$ is multiplicity free and

$$
\tau_{n}^{\lambda}=\bigoplus_{\lambda \succ \lambda^{1}} \tau_{n-1}^{\lambda^{1}}
$$

where $\lambda^{1}=\left(\lambda_{1}^{1}, \ldots, \lambda_{[(n-1) / 2]}^{1}\right) \in \Lambda(n-1, e)$ and $\lambda \succ \lambda^{1}$ is defined as follows. There are two cases:

- If $n$ is odd then $[n / 2]=(n-1) / 2$ and $\lambda \succ \lambda^{1}$ is defined by

$$
\lambda_{1} \geq \lambda_{1}^{1} \geq \lambda_{2} \geq \lambda_{2}^{1} \geq \ldots \geq \lambda_{(n-1) / 2]} \geq \lambda_{(n-1) / 2}^{1} \geq-\lambda_{(n-1) / 2} .
$$

- If $n$ is even, then $\lambda \succ \lambda^{1}$ is defined by

$$
\lambda_{1} \geq \lambda_{1}^{1} \geq \lambda_{2} \geq \lambda_{2}^{1} \geq \ldots \geq \lambda_{n / 2-1} \geq \lambda_{n / 2-1}^{1} \geq\left|\lambda_{n / 2}\right| .
$$

Let $\mathfrak{s o}(i)$ be the subalgebra of $\mathfrak{s o}(n)$ spanned by $I_{k l}$ for all $k, l \leq i$. Consider the chain of decreasing Lie subalgebras $\mathfrak{s o}(n) \supset \mathfrak{s o}(n-1) \supset \ldots \supset \mathfrak{s o}(2)$. By restricting $\tau_{n}^{\lambda}$ successively to these subalgebras, $\tau_{n}^{\lambda}$ becomes a direct sum of 1 dimensional irreducible representations of $\mathfrak{s o}(2)$. By choosing a unit vector from each of these 1 dimensional subspaces, we have an 
orthonormal basis of $\tau_{n}^{\lambda}$ consisting of unit vectors called the Gelfand-Zetlin basis. Thus, each basis vector is represented by an array

$$
M=\left(\lambda, \lambda^{1}, \ldots, \lambda^{n-2}\right)
$$

where $\lambda^{i} \in \Lambda(n-i, e)$ and $\lambda_{i} \succ \lambda_{i+1}$. The corresponding unit vector will be denoted by $\mathbf{v}_{M}$. Note that this vector generates the irreducible representation $\tau_{n-i}^{\lambda^{i}}$ under the action of $\mathfrak{s o}(n-i)$.

An explicit formula of $I_{i+1, i} \cdot \mathbf{v}_{M}$ as a linear combination of vectors in the Gelfand-Zetlin basis is given on page 364 in [VK].

Induced representations of $\operatorname{Spin}(n, 1)$. Let $\mu \in \Lambda(n-1)$ and let $\operatorname{Ind}_{n, 1}(\mu, c)$ denote the Harish-Chandra module of the normalized induced representation

$$
\operatorname{Ind}_{\operatorname{Spin}(n-1) \cdot \mathbb{R}^{+} \cdot N}^{\operatorname{Spin}(n, 1)}\left(\tau_{n-1}^{\mu} \otimes \exp (c) \otimes 1\right) .
$$

We will equip $\operatorname{Ind}_{n, 1}(\mu, c)$ with an Hermitian form given by

$$
\int_{\operatorname{Spin}(n)} f_{1}(k) \overline{f_{2}(k)} d k
$$

for $f_{1}, f_{2} \in \operatorname{Ind}_{n, 1}(\mu, c)$ and $d k$ is the Haar measure on $\operatorname{Spin}(n)$ with with the total volume 1 .

We note that $\operatorname{Ind}_{n, 1}(\mu, c)$ is multiplicity free as a representation of $\operatorname{Spin}(n)$. Indeed

$$
\operatorname{Ind}_{n, 1}(\mu, c)=\bigoplus_{\lambda \succ \mu} \tau_{n}^{\lambda}
$$

By the above discussion and (5), we can assign an orthonormal Gelfand-Zetlin basis to each $\tau_{n}^{\lambda}$. Thus, $\operatorname{Ind}_{n, 1}(\mu, c)$ has a basis consisting of vectors $\mathbf{v}_{M}$ one for every array $M$ as in (5) starting with $\lambda \succ \mu$. This basis is denoted by $B_{\mu}$. We shall assume that this basis is orthonormal under the Hermitian form in (6).

Hirai $[\mathrm{Hi}]$ and Klimyk $[\mathrm{KG}]$ determined all the irreducible subquotients of $\operatorname{Ind}_{n, 1}(\mu, c)$ with the help of the orthonormal basis above. They also obtained a classification of the unitary dual of $\operatorname{Spin}(n, 1)$. In the rest of this section, we will reproduce some of their results below which we will need later. These results are entirely due to or easy consequences of $[\mathrm{Hi}]$ and $[\mathrm{KG}]$.

First, we shall give an explicit action of the Lie algebra $\mathfrak{s o}(n, 1)$ on the induced representations. To that end, we identify the Lie algebra $\mathfrak{s o}(n, 1)$ with the subspace of $(n+1) \times(n+1)$ real matrices spanned by $I_{k l}$ for $l<k \leq n$ and

$$
J_{n+1, i}=E_{i, n+1}+E_{n+1, i} .
$$

The Lie algebra $\mathfrak{s o}(n, 1)$ is generated by $I_{i+1, i}(i<n)$ and $J_{n+1, n}$. The elements $I_{i+1, i}$ $(i<n)$ preserve $\operatorname{Spin}(n)$-types, and the action is given on page 364 in [VK]. The most interesting, of course, is the action of $J_{n+1, n}$ which is given as follows. Let $\mathbf{v}_{M}$ be in $B_{\mu}$. 
Let $M^{+s}$ (resp. $M^{-s}$ ) denote the array obtained from $M$ by increasing (resp. decreasing) $\lambda_{s}$ by 1 . Then

$$
J_{n+1, n} \mathbf{v}_{M}=\sum_{s=1}^{\left[\frac{n}{2}\right]} \omega_{s}(c, \mu, M) \mathbf{v}_{M^{+s}}-\sum_{s=1}^{\left[\frac{n}{2}\right]} \omega_{s}\left(-c, \mu, M^{-s}\right) \mathbf{v}_{M^{-s}}+c \omega_{0}(\mu, M) \mathbf{v}_{M}
$$

where

$$
\omega_{s}(c, \mu, M)=\left(\lambda_{s}+\frac{n+1}{2}-s+c\right) \omega_{s}(\mu, M)
$$

and $\omega_{s}(\mu, M)$ is defined on pages 418-419 in [VK]. It depends on $\mu$ and the first two rows $\lambda \succ \lambda^{1}$ of $M$ only. Also $\omega_{0}(\mu, M)=0$ if $n$ is even. If $n$ is odd then

$$
\omega_{0}=\prod_{i=1}^{\frac{n-1}{2}} \frac{\left(\mu_{i}+\frac{n-1}{2}-i\right)\left(\lambda_{i}^{1}+\frac{n-1}{2}-i\right)}{\left(\lambda_{i}+\frac{n+1}{2}-i\right)\left(\lambda_{i}+\frac{n-1}{2}-i\right)} .
$$

Note that $\omega_{0}(\mu, M) \neq 0$ if $\mu$ is half integral. This observation is crucial in the proof of uniqueness of $V$. More precisely, consider the Cartan decomposition $\mathfrak{s o}(n) \oplus \mathfrak{p}_{0}$ of $\mathfrak{s o}(n, 1)$. Let $\Pi$ be an $(\mathfrak{s o}(n, 1)$, $\operatorname{Spin}(n))$-module with multiplicity free $\operatorname{Spin}(n)$-types. This situation occurs for all induced representations and, therefore, all irreducible $(\mathfrak{s o}(n, 1), \operatorname{Spin}(n))$ modules. Let $\tau^{\lambda}$ a $\operatorname{Spin}(n)$-type in $\Pi$. Since $\mathfrak{p}_{0} \otimes \mathbb{C} \cong \mathbb{C}^{n}$, the action of $\mathfrak{p}_{0}$ on $\tau^{\lambda}$ followed by the projection on $\tau^{\lambda}$ defines a $\operatorname{Spin}(n)$-equivariant map

$$
p_{\lambda}: \mathbb{C}^{n} \otimes \tau^{\lambda} \rightarrow \tau^{\lambda} .
$$

If $n$ is even then $\mathbb{C}^{n}$, the standard representation of $\mathfrak{s o}(n)$, does not contain a trivial weight. Therefore the tensor product $\mathbb{C}^{n} \otimes \tau^{\lambda}$ does not contain $\tau^{\lambda}$. This shows that $p_{\lambda}$ is always zero if $n$ is even. In general, $p_{\lambda}$ depends on the action of $J_{n+1, n}$. Uniqueness of $V$ is based on the following.

Proposition 3.1. Assume that $n$ is odd. Let $\Pi$ be an irreducible $(\mathfrak{s o}(n, 1)$, $\operatorname{Spin}(n))$ module with half-integral types. Assume that $p_{\lambda}=0$ for every $\operatorname{Spin}(n)$-type $\tau^{\lambda}$ of $\Pi$. Then $\Pi$ is isomorphic to $\operatorname{Ind}_{n, 1}(\mu, 0)$ for some $\mu$ in $\Lambda\left(\frac{n-1}{2}, \frac{1}{2}\right)$. In particular, $\Pi$ is determined by its minimal $\operatorname{Spin}(n)$-type.

Proof. Suppose $\Pi$ is a subquotient of $\operatorname{Ind}(\mu, c)$ for some $\mu$ in $\Lambda\left(\left[\frac{n-1}{2}\right], \frac{1}{2}\right)$. The map $p_{\lambda}$ is determined by the action of the operator $J_{n+1, n}$. Since $\mu$ is half-integral, as we remarked above, $\omega_{0}(\mu, M) \neq 0$ for all $M$. This shows that $p_{\lambda} \neq 0$ for all types of the induced representation unless $c=0$. This shows that $\Pi$ is contained in $\operatorname{Ind}(\mu, 0)$ for some $\mu$. Since $\operatorname{Ind}(\mu, 0)$ is irreducible and determined by its minimal type among all induced representations with $c=0$, the proposition follows.

Discrete series. The group $\operatorname{Spin}(n, 1)$ has square integrable representations if and only if $n$ is even. Let $\mu \in \Lambda(n-1, e)$. Suppose that $c+e+\frac{1}{2}$ is a positive integer. Then 
$\operatorname{Ind}_{n, 1}(\mu, c)$ contains two discrete series representations (or limits of discrete series if $c=0$ and $\left.e=\frac{1}{2}\right)$ with $\operatorname{Spin}(n)$-types

$$
D^{+}=\bigoplus_{\lambda_{\frac{n}{2}} \geq c^{\prime}} \tau_{n}^{\lambda} \text { and } D^{-}=\bigoplus_{\lambda_{\frac{n}{2}} \leq-c^{\prime}} \tau_{n}^{\lambda}
$$

where $c^{\prime}=c+\frac{1}{2}$.

Remark. We would like to point out three errors in [VK]. The right hand side of Eq. (6) on page 418 should be divided by 2 . The factor $\left(4 l_{s, 2 k+1}-1\right)$ in the denominator on the right hand side of Eq. (8) is incorrect. It should be $\left(4 l_{s, 2 k+1}^{2}-1\right)$. The first line of page 419 should be $l_{i}=r_{i}+\left[\frac{n-1}{2}\right]-i$. Also see page 86 in [Hi] for the correct formulas.

\section{UNIQUENESS}

In this section we show that the $K$-modules introduced in Section 2 can be extended to $(\mathfrak{g}, K)$-modules in at most one way. Moreover, the extension is necessarily irreducible and unitarizable.

Let $U$ denote the real vector space with basis $\left\{u_{1}, \ldots, u_{p+q}\right\}$. We equip $U$ with a symmetric bilinear form B of signature $(p, q)$ such that

$$
\mathrm{B}\left(u_{i}, u_{j}\right)=\delta_{i j} \epsilon_{i}
$$

where $\epsilon_{i}=1$ if $i \leq p$ and $\epsilon_{i}=-1$ if $i>p$. We realize $\mathfrak{s o}(p, q)$ as $(p+q) \times(p+q)$-matrices skew symmetric with respect to the bilinear form B. Let $E_{i j}$ denote the $(p+q) \times(p+q)$ square matrix whose $(i, j)$-th entry is 1 and 0 elsewhere. Of special interest will be the following elements in $\mathfrak{s o}(p, q)$ :

$$
\left\{\begin{array}{l}
I_{i+1, i}=E_{i, i+1}-E_{i+1, i} \text { for } i \neq p, \\
J_{p+1, p}=E_{p, p+1}+E_{p+1, p} .
\end{array}\right.
$$

For $i=1,2 \ldots$ let $U_{i}$ denote the subspace of $U$ spanned by $\left\{u_{1}, \ldots, u_{i}\right\}$. Let $q=a+b$ for some non-negative integers $a$ and $b$. Then the stabilizer of $U_{p+a}$ is

$$
\mathfrak{s o}(p, a) \oplus \mathfrak{s o}(b) .
$$

These algebras are of special interest to us. We pick $K$ so that its Lie algebra is the stabilizer of $U_{p}$. We also note that the subalgebra $\mathfrak{s o}(p, 1)$ (case $a=1$ ) is generated by $\mathfrak{s o}(p)$ and $J_{p+1, p}$.

Case of $V$.

Proposition 4.1. Assume that the $K$-module $V$ extends to a $\mathfrak{s o}(p, q)$-module. Then this extension is unique, irreducible and unitarizable. Moreover, the restriction of $V$ to $\mathfrak{s o}(p, 1) \times \mathrm{SO}_{q-1}$ decomposes discretely as a direct sum

$$
\bigoplus_{\lambda \in \Lambda(p, 0)} \operatorname{Ind}_{p, 1}\left(\lambda+\frac{q-p}{2} \mathbf{1}_{\frac{p-1}{2}}, 0\right) \otimes \tau_{q-1}^{\left(\lambda_{1}, \ldots, \lambda_{\frac{p-1}{2}}, 0, \ldots, 0\right)} .
$$


Proof. Recall that

$$
V=\bigoplus_{\lambda \in \Lambda(p, 0)} \tau_{p}^{A(\lambda)} \otimes \tau_{q}^{B(\lambda)}
$$

where, for $\lambda=\left(\lambda_{1}, \ldots, \lambda_{\frac{p-1}{2}}\right) \in \Lambda(p, 0), A(\lambda)=\lambda+\frac{q-p}{2} \mathbf{1}_{\frac{p-1}{2}}$ and $B(\lambda)=\left(\lambda, \mathbf{0}_{\frac{q-p+1}{2}}\right)$.

As in the previous section, let $\left\{\mathbf{v}_{M}\right\}$ be an orthonormal Gelfand-Zetlin basis of $\tau_{p}^{A(\lambda)}$ defined with respect to the chain of subgroups $\mathfrak{s o}\left(U_{p}\right) \supseteq \mathfrak{s o}\left(U_{p-1}\right) \supseteq \ldots$. Similarly let $U_{p}^{c}$ denote the orthogonal complement of $U_{p}$ and let $\left\{\mathbf{v}_{N}\right\}$ be an orthonormal Gelfand-Zetlin basis of $\tau_{q}^{B(\lambda)}$ defined with respect to the chain of subgroups $\mathfrak{s o}\left(U_{p}^{c}\right) \supseteq \mathfrak{s o}\left(U_{p+1}^{c}\right) \supseteq \ldots$. It follows that

$$
\mathbf{v}_{M} \otimes \mathbf{v}_{N}
$$

where $M$ and $N$ are arrays with the first row $A(\lambda)$ and $B(\lambda)$ respectively, is a basis of $V$. Since $\mathfrak{s o}(p, q)$ is generated by $\mathfrak{s o}(p) \oplus \mathfrak{s o}(q)$ and $J_{p+1, p}$ it suffices to show that $J_{p+1, p}$ acts in only one possible way on the basis $\mathbf{v}_{M} \otimes \mathbf{v}_{N}$.

Note that $J_{p+1, p}$ and $\mathfrak{s o}(p)$ generate $\mathfrak{s o}(p, 1)$. Define an equivalence relation on the basis vectors of $V$ by $\mathbf{v}_{M} \otimes \mathbf{v}_{N} \sim \mathbf{v}_{M^{\prime}} \otimes \mathbf{v}_{N^{\prime}}$ if the arrays $N$ and $N^{\prime}$ have all rows equal except, perhaps, the first row. Fix an equivalence class $C$. Let $\Pi$ be the subspace of $V$ spanned by all basis vectors in $C$. If $V$ extends to a $\mathfrak{s o}(p, q)$-module, then $\Pi$ is an $(\mathfrak{s o}(p, 1), \operatorname{Spin}(p))$ module. Its $\operatorname{Spin}(p)$-types are described as follows. Let $\left(y_{1}, \ldots, y_{\frac{p-1}{2}}, 0 \ldots, 0\right)$ be the second row of the array $N$ for all basis vectors in the equivalence class $C$. Define

$$
\mu=\left(y_{1}, \ldots, y_{\frac{p-1}{2}}\right)+\frac{1}{2}(q-p) \mathbf{1}_{\frac{p-1}{2}} \in \Lambda\left(p-1, \frac{1}{2}\right) .
$$

Notice that $A(\lambda) \succ \mu$. In fact, the $\operatorname{Spin}(p)$-types of $\Pi$ are the same as the types of the induced representation $\operatorname{Ind}_{p, 1}(\mu, c)$. We claim that

$$
\Pi \cong \operatorname{Ind}_{p, 1}(\mu, 0) \text {. }
$$

In view of Proposition 3.1 we must show that $p_{A(\lambda)}=0$ for any type $\tau^{A(\lambda)}$ of $\Pi$. In other words, we must show that the action of $J_{p+1, p}$ on $\tau^{A(\lambda)}$ composed with the projection on $\tau^{A(\lambda)}$ is zero. But this is easy. Indeed, the maximal $\tau^{A(\lambda)}$-isotypic summand of $V$ is given by

$$
\tau^{A(\lambda)} \otimes \tau^{B(\lambda)}
$$

The action of $J_{p+1, p}$ is a part of the action of $\mathfrak{p}_{0} \otimes \mathbb{C} \cong \mathbb{C}^{p} \otimes \mathbb{C}^{q}$ (of the Cartan decomposition of $\mathfrak{s o}(p, q))$. Since $q$ is even, $\mathbb{C}^{q} \otimes \tau^{B(\lambda)}$ does not contain $\tau^{B(\lambda)}$ as a summand. This shows that $p_{A(\lambda)}=0$ and $\Pi$ must be isomorphic to $\operatorname{Ind}_{p, 1}(\mu, 0)$. Let $T: \operatorname{Ind}_{p, 1}(\mu, 0) \rightarrow \Pi$ be an isomorphism. In particular, the action of $J_{p+1, p}$ must be equal to $T \circ \pi\left(J_{p+1, p}\right) \circ T^{-1}$ where $\pi\left(J_{p+1, p}\right)$ is the action of $J_{p+1, p}$ on $\operatorname{Ind}_{p, 1}(\mu, 0)$. Since $T$ is unique up to a non-zero scalar the action of $\mathfrak{s o}(p, q)$ on $V$ is unique.

Next we show that $V$ is unitarizable. Let $\bar{V}$ denote the Hermitian dual of $V$. Since it has the same $K$-type as $V$, it follows that $\bar{V}$ and $V$ are isomorphic $(\mathfrak{s o}(p, q), K)$-modules. This isomorphism induces a non-degenerate $(\mathfrak{s o}(p, q), K)$-invariant Hermitian form on $V$. 
We may assume that it is positive definite on the minimal $K$-type. We claim that the Hermitian form is positive definite so $V$ is unitarizable. Let $\tau_{p, q}^{\lambda}:=\tau_{p}^{A(\lambda)} \otimes \tau_{q}^{B(\lambda)}$ where $\lambda=\left(\lambda_{1}, \ldots, \lambda_{\frac{p-1}{2}}\right)$. Suppose $\tau_{p, q}^{\lambda-\varepsilon_{s}}$ is nonzero and the signatures on $\tau_{p, q}^{\lambda}$ and $\tau_{p, q}^{\lambda-\varepsilon_{s}}$ are different. Define

$$
\mu^{\prime}=\left(\lambda_{1}, \ldots, \lambda_{\frac{p-1}{2}}\right)-\varepsilon_{s}+\frac{q-p}{2} \mathbf{1}_{\frac{p-1}{2}} \in \Lambda(p-1)
$$

and $\Pi^{\prime}=\operatorname{Ind}_{p, 1}\left(\mu^{\prime}, 0\right)$. Then $\Pi^{\prime}$ intersects the two $K$-types $\tau_{p, q}^{\lambda}$ and $\tau_{p, q}^{\lambda-\varepsilon_{s}}$ non-trivially. The restriction of the invariant Hermitian form of $V$ to $\Pi^{\prime}$ is positive definite since $\Pi^{\prime}$ is unitarizable. This contradicts the fact that the signatures are different on $\tau_{p, q}^{\lambda}$ and $\tau_{p, q}^{\lambda-\varepsilon_{s}}$ and proves our claim.

Finally we show that $V$ is irreducible. Suppose $W$ is a proper submodule of $V$. By taking its orthogonal complement if necessary, we assume that $W$ does not contain the minimal $K$-type. Let $\tau_{p, q}^{\lambda}=\tau_{p}^{A(\lambda)} \otimes \tau_{q}^{B(\lambda)}$ be a $K$-type in $W$ such that $\sum_{i} \lambda_{i}$ is minimal. Since $\tau_{p, q}^{\lambda}$ is not the minimal $K$-type, $\tau_{p, q}^{\lambda-\varepsilon_{s}}$ is nonzero for some $s \leq \frac{p-1}{2}$. We define $\mu^{\prime}$ and $\Pi^{\prime}=\operatorname{Ind}_{p, 1}\left(\mu^{\prime}, 0\right)$ as in (11). Then $\Pi^{\prime}$ intersects $\tau_{p, q}^{\lambda}$ and $\tau_{p, q}^{\lambda-\varepsilon_{s}}$ non-trivially. Hence $W$ contains $\tau_{p, q}^{\lambda-\varepsilon_{s}}$. This contradicts that fact that $\sum_{i} \lambda_{i}$ is minimal in $W$. Therefore $V$ is irreducible and this completes the proof of the proposition.

By scaling the basis vectors $\mathbf{v}_{M} \otimes \mathbf{v}_{N}$ in (8) if necessary, we may assume that the basis vectors they form an orthonormal basis of $V$ and, the action of $J_{p+1, p}$ on $\Pi$ in (10) is the same as the action of $J_{p+1, p}$ on the basis vectors $B_{\mu}$ of the induced representation in (7):

$$
\begin{aligned}
J_{p+1, p} \mathbf{v}_{M} \otimes \mathbf{v}_{N} & =\sum_{s=1}^{\frac{p-1}{2}}\left(\lambda_{s}+\frac{q+1}{2}-s\right) \omega_{s}(\mu, M) \mathbf{v}_{M^{+s}} \otimes \mathbf{v}_{N^{+s}} \\
& -\sum_{s=1}^{\frac{p-1}{2}}\left(\lambda_{s}+\frac{q-1}{2}-s\right) \omega_{s}\left(\mu, M^{-s}\right) \mathbf{v}_{M^{-s}} \otimes \mathbf{v}_{N^{-s}} .
\end{aligned}
$$

Case of $V_{o}^{ \pm}$. We remind the reader that $q=p-1$ holds here.

Proposition 4.2. Assume that $V_{o}^{+}$(respectively $V_{o}^{-}$) extends to a $\mathfrak{s o}(p, p-1)$-module. Then this extension is unique, irreducible and unitarizable. Moreover, the restriction of $V_{o}^{ \pm}$to $\mathfrak{s o}(p, 1) \times \mathrm{SO}(p-2)$ decomposes discretely as a direct sum

$$
\bigoplus_{\lambda \in \Lambda(p-2,0), \lambda_{\frac{p-3}{2}} \geq 1} \operatorname{Ind}_{p, 1}\left((\lambda, 1)-\frac{1}{2} \mathbf{1}_{\frac{p-1}{2}}, 0\right) \otimes \tau_{p-2}^{\lambda} .
$$

The proof is identical to one of Proposition 4.1, so it is omitted.

Case of $V^{ \pm}$. Since $V^{+}$and $V^{-}$, as $(\mathfrak{s o}(p, q), K)$-modules have been constructed by Knapp in $[\mathrm{Kn}]$, here we describe a somewhat different result needed to construct $V, V_{o}^{+}$and $V_{o}^{-}$ via the Wallach transfer of $V^{+}$and $V^{-}$. 
Proposition 4.3. The representation $V^{+}\left(\right.$resp. $\left.V^{-}\right)$is the unique representation with $K$ types as in (4). The restriction of $V^{+}$(respectively $\left.V^{-}\right)$to $\mathrm{SO}(p-1) \times \mathfrak{s o}(q, 1)$ decomposes discretely as a direct sum

$$
\bigoplus_{\lambda \in \Lambda(p-1,0), \lambda \frac{q}{2}+1} \tau_{p-1}^{\lambda} \otimes \Theta(\lambda)
$$

where $\Theta(\lambda)$ is the discrete series representation of $\operatorname{Spin}(q, 1)$ (or limit of discrete series if $p-1=q$ and $\left.\lambda_{\frac{q}{2}}=0\right)$ whose minimal $\operatorname{Spin}(q)$-type has highest weight $\left(\lambda_{1}, \ldots, \lambda_{\frac{q}{2}}\right)+\frac{p-q}{2} \mathbf{1}_{\frac{q}{2}}$ (respectively $\left.\sigma\left(\left(\lambda_{1}, \ldots, \lambda_{\frac{q}{2}}\right)+\frac{p-q}{2} \mathbf{1}_{\frac{q}{2}}\right)\right)$ and the infinitesimal character

$$
\left(\lambda_{1}, \ldots, \lambda_{\frac{q}{2}}\right)+\frac{p-q}{2} \mathbf{1}_{\frac{q}{2}}+\rho_{q+1} .
$$

\section{EXISTENCE}

Since $\mathfrak{s o}(p, q)$ is generated by $\mathfrak{s o}(p, 1)$ and $\mathfrak{s o}(q)$, the formula for $J_{p+1, p}$ in (12) gives a representation of $\mathfrak{s o}(p, q)$ on $V$, provided that certain relations have been verified. The same also applies to $V^{ \pm}$and $V_{o}^{ \pm}$. While the verification is straightforward, it is also rather cumbersome. It is much quicker to construct $V$ and $V_{o}^{ \pm}$from $V^{ \pm}$using the Wallach transfer.

If $p-1 \geq q$ then Knapp [Kn] has constructed two representations with the same $K$ types as $V^{+}$and $V^{-}$. Trapa [T] has further established some properties of $V^{+}$and $V^{-}$. In order to state their results, define

$$
\mu_{p, q}=\left\{\begin{array}{l}
\left(\frac{p-1}{2}, \frac{p-3}{2}, \ldots, 1, \frac{q-1}{2}, \frac{q-3}{2}, \ldots, \frac{1}{2}\right) \text { if } p<q-1 \\
\left(\frac{q}{2}, \frac{q-2}{2}, \ldots, 1, \frac{p-2}{2}, \frac{p-4}{2}, \ldots, \frac{1}{2}\right) \text { if } p \geq q-1
\end{array}\right.
$$

Theorem 5.1. If $p-1 \geq q$ then $V^{+}$and $V^{-}$can be extended to unitarizable and irreducible $(\mathfrak{s o}(p, q), K)$-modules. The infinitesimal character of $V^{+}$and $V^{-}$is $\mu_{p, q}$ and the annihilator in the universal algebra is the unique maximal two sided ideal with the infinitesimal character $\mu_{p, q}$.

We can now use the Wallach transfer to construct $V$ from $V^{ \pm}$. Take first $p-1<$ q. Consider $V^{ \pm}$for $\mathfrak{s o}(q+1, p-1)$. The restriction of $V^{ \pm}$to $\mathrm{SO}(q) \times \mathfrak{s o}(p-1,1)$ is given by Proposition 4.3. Let $\Gamma_{\mathfrak{s o}(p, 1)}^{i}$ denote the $i$-th derivative of the Zuckerman functor with respect to $\mathfrak{s o}(p, 1)$. Then, by [Wa1], $\Gamma_{\mathfrak{s o}(p, 1)}^{\frac{p-1}{2}}$ applied to a discrete series representation produces an irreducible finite dimensional representation with the same infinitesimal character. It follows that the $\mathfrak{s o}(p, q)$-modules

$$
\Gamma_{\mathfrak{s o}(p-1,1)}^{\frac{p-1}{2}}\left(V^{+}\right) \text {and } \Gamma_{\mathfrak{s o}(p-1,1)}^{\frac{p-1}{2}}\left(V^{-}\right)
$$

have the same types as $V$. By uniqueness of $V$ in Proposition 4.1, these two representations must be isomorphic. Since Zuckerman's functor can only increase the annihilator, we have the following theorem: 
Theorem 5.2. If $p-1<q$ then $V$ can be extended to unitarizable and irreducible $(\mathfrak{s o}(p, q), K)$-module. The infinitesimal character of $V$ is $\mu_{p, q}$ and the annihilator in the universal algebra is the unique maximal two sided ideal with the infinitesimal character $\mu_{p, q}$.

Finally, assume that $p-1=q$. Consider $V^{ \pm}$for $\mathfrak{s o}(p, p-1)$. The restriction of $V^{ \pm}$to $\mathrm{SO}(p-1) \times \mathfrak{s o}(p-1,1)$ is given by Proposition 4.3. Then, by [Wa1], $\mathfrak{s o}(p, p-1)$-modules

$$
\Gamma_{\mathfrak{s o}(p-1,1)}^{\frac{p-1}{2}}\left(V^{+}\right) \text {and } \Gamma_{\mathfrak{s o}(p-1,1)}^{\frac{p-1}{2}}\left(V^{-}\right)
$$

have the same types as $V_{o}^{+}$and $V_{o}^{-}$, respectively. Again, since Zuckerman's functor can only increase the annihilator, we have the following theorem:

Theorem 5.3. If $p-1=q$ then $V_{o}^{ \pm}$can be extended to unitarizable and irreducible $(\mathfrak{s o}(p, p-1), K)$-modules. The infinitesimal character of $V_{o}^{ \pm}$is $\mu_{p, p-1}$ and the annihilator in the universal algebra is the unique maximal two sided ideal with the infinitesimal character $\mu_{p, p-1}$.

\section{Associated VARiety}

In this section we will compute the associated varieties of $V, V^{ \pm}$and $V_{o}^{ \pm}$. A definition and basic properties of associated varieties could be found in [Vo2]. In order to simplify notation, define

$$
m=\min \left(\frac{p-1}{2}, \frac{q}{2}\right) .
$$

Recall that nilpotent orbits of the complex group $\mathrm{O}_{n}(\mathbb{C})$ are parameterized by partitions of $n$ such that every even part has an even multiplicity. The classification of (real) nilpotent $\mathrm{O}(p, q)$-orbits on $\mathfrak{s o}(p, q)$ is refined as follows: To every partition we attach the Young diagram as usual. Then we insert signs + and - into the boxes corresponding to odd (length) rows such that the signs alternate. Then this signed partition parameterizes an orbit of $\mathrm{O}(p, q)$ if and only if the difference of the number of positive and negative signs is equal to the signature $p-q$. Two signed partitions correspond to the same real orbit if and only if one signed partition can be obtained form another by permuting the rows of the same length.

Case $p-1<q$. Consider the partition $\left(2^{p-1}, 1^{q-p+2}\right)$. The number of odd rows is $q-p+2$. On the other hand, the signature is equal to $p-q$. Thus we can mark the Young diagram by putting + in the first row of length 1 and - in all other. Let $\mathcal{O}_{2^{p-1}}$ be the corresponding nilpotent $\mathrm{O}(p, q)$-orbit. Let $\mathfrak{g}=\mathfrak{k} \oplus \mathfrak{p}$ denote the complexified Cartan decomposition

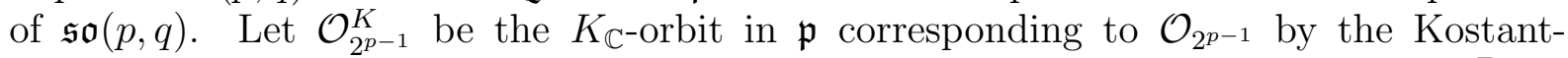
Sekiguchi correspondence. We shall now describe this orbit in more details. Let $\bar{K}_{\mathbb{C}}=$ $\mathrm{SO}_{p}(\mathbb{C}) \times \mathrm{SO}_{q}(\mathbb{C})$. Recall that $\mathfrak{p} \cong \mathbb{C}^{p} \otimes \mathbb{C}^{q}$ under the action of $\bar{K}_{\mathbb{C}}$. Let $($,$) denote a$ $\mathrm{SO}_{p}(\mathbb{C})$-invariant symmetric bilinear form on $\mathbb{C}^{p}$. Pick a basis $\left\{\mathbf{e}_{1}, \ldots, \mathbf{e}_{p}\right\}$ of $\mathbb{C}^{p}$ so that $\left(\mathbf{e}_{i}, \mathbf{e}_{j}\right)=0$ except $\left(\mathbf{e}_{i}, \mathbf{e}_{p+1-i}\right)=1$. We do likewise for $\mathbb{C}^{q}$ to get a basis $\left\{\mathbf{f}_{1}, \ldots, \mathbf{f}_{q}\right\}$. 
Using these two bases, we can identify $\mathfrak{p}$ with the set of $p$ by $q$ matrices so that the $\mathbf{e}_{i} \otimes \mathbf{f}_{j}$ corresponds to the elementary matrix $E_{i, j}$. Define

$$
P=E_{1,1}+E_{2,2}+\cdots+E_{m, m} \in \mathfrak{p} .
$$

Let $\iota$ be the standard representation of $\mathfrak{s o}_{p+q}(\mathbb{C})$. Then it is a simple exercise to show that

- the null space of $\iota(P)$ has the dimension $q+1$.

- $\iota(P)^{2}=0$, that is, $\iota(P)$ is a nilpotent element.

This implies that $P$ belongs to the complex nilpotent $\mathrm{O}_{p+q}(\mathbb{C})$-orbit corresponding to the partition $\left(2^{p-1}, 1^{q-p+2}\right)$. Thus, the orbit $\mathcal{O}_{2^{p-1}}^{K}$ is generated by $P$.

For the rest of this section, we identify $\mathfrak{p}$ with $\mathfrak{p}^{*}$ using the Killing form, and we identify adjoint orbits with co-adjoint orbits.

Let $U_{n}(\mathfrak{g})$ denote the standard filtration on the universal enveloping algebra of $\mathfrak{s o}(p, q)$. Let $F_{n}=U_{n}(\mathfrak{g}) \cdot \tau_{\text {min }}$ be the subspace in $V$ where $\tau_{\text {min }}=\tau_{p}^{\frac{q-p}{2}} \mathbf{1}_{m} \otimes \mathbb{C}$ is the minimal $K$-type of $V$. The graded module

$$
\operatorname{Gr}(V)=\bigoplus_{n=0}^{\infty} F_{n} / F_{n-1}
$$

is a $\left(\operatorname{Sym}(\mathfrak{p}), K_{\mathbb{C}}\right)$-module generated by $\tau_{\text {min }}=F_{0}$. Note that $\mathfrak{k} \cdot F_{n} \subseteq F_{n}$ so $\mathfrak{k}$ acts trivially on the graded module. By induction we have $\mathfrak{p} \cdot F_{n}=F_{n+1}$. By the formula $J_{p+1, p} \in \mathfrak{p}$ on the $K$-types of $V$ in (12), we see that

$$
\mathrm{Gr}_{n}(V)=F_{n} / F_{n-1}=\bigoplus_{\lambda} \tau_{p}^{\lambda+\frac{q-p}{2} \mathbf{1}_{m}} \otimes \tau_{q}^{(\lambda, \mathbf{0})}
$$

where the sum is taken over $\lambda=\left(\lambda_{1}, \ldots, \lambda_{m}\right) \in \Lambda(p, 0)$ such that $\sum_{i} \lambda_{i}=n$. Let $I$ be the annihilator ideal of $\operatorname{Gr}(V)$ in $\operatorname{Sym}(\mathfrak{p})$. It is also the annihilator ideal of $\tau_{\min }=\operatorname{Gr}_{0}(V)$. The variety in $\mathfrak{p}^{*} \cong \mathfrak{p}$ cut out by $I$ is called the associated variety of $V$. We now state the main theorem of this section.

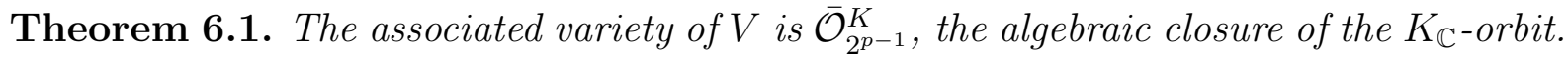
More precisely,

$$
\operatorname{Sym}(\mathfrak{p}) / I=\Gamma\left(\overline{\mathcal{O}}_{2^{p-1}}^{K}\right),
$$

the ring of regular functions on $\overline{\mathcal{O}}_{2^{p-1}}^{K}$.

Proof. The first step in the proof is a description of the space of regular functions on $\overline{\mathcal{O}}_{2^{p-1}}^{K}$ as a $K_{\mathbb{C}}$-module.

Lemma 6.2. Let $\Gamma\left(\overline{\mathcal{O}}_{2^{p-1}}^{K}\right)$ be the algebra of regular functions on the closure $\overline{\mathcal{O}}_{2^{p-1}}^{K}$ of $\mathcal{O}_{2^{p-1}}^{K}$. We have the following isomorphism of $\bar{K}_{\mathbb{C}}=\mathrm{SO}_{p} \times \mathrm{SO}_{q}$-modules

$$
\Gamma\left(\overline{\mathcal{O}}_{2^{p-1}}^{K}\right)=\bigoplus_{\lambda \in \Lambda(p, 0)} \tau_{p}^{\lambda} \otimes \tau_{q}^{(\lambda, \mathbf{0})}
$$


Proof. Recall that we have identified $\mathfrak{p}$ with the set of complex $p \times q$ matrices. Consider the $m \times m$ block located in the upper left corner. The stabilizer of this block in $\mathrm{SO}_{p} \times \mathrm{SO}_{q}$ is a product of two maximal parabolic subgroups $Q_{1}=L_{1} N_{1}$ and $Q_{2}=L_{2} N_{2}$ with Levi factors

$$
L_{1}=\mathrm{GL}_{m} \text { and } L_{2}=\mathrm{GL}_{m} \times \mathrm{SO}_{q-2 m}
$$

Since $P$ is the identity matrix located in the $m \times m$ block, it is now clear that the stabilizer of $P$ in $\bar{K}_{\mathbb{C}}$ is

$$
\bar{K}_{\mathbb{C}}(P)=\triangle \mathrm{GL}_{m} \times N_{1} \times \mathrm{SO}_{q-2 m} \times N_{2}
$$

where $\triangle \mathrm{GL}_{m}=\mathrm{GL}_{m}$ is diagonally embedded in $\mathrm{GL}_{m} \times \mathrm{GL}_{m} \subseteq L_{1} \times L_{2}$. It follows that every regular function on $\overline{\mathcal{O}}_{2^{p-1}}^{K}$ gives a right $\bar{K}_{\mathbb{C}}(P)$-invariant regular function on $\bar{K}_{\mathbb{C}}$. The Peter-Weyl Theorem implies that the subspace of $\bar{K}_{\mathbb{C}}(P)$-invariant functions on $\bar{K}_{\mathbb{C}}$ has precisely the types given by the right hand side of $(14)$. This implies that $\Gamma\left(\overline{\mathcal{O}}_{2^{p-1}}^{K}\right)$ is contained in the right hand side of (14)

To prove the opposite inclusion, we consider $\operatorname{Sym}(\mathfrak{p})$ as a $\mathrm{GL}_{p} \times \mathrm{GL}_{q}$-module, since $\mathfrak{p}$ has been identified with the set of $p \times q$-matrices. Then

$$
\operatorname{Sym}(\mathfrak{p})=\bigoplus_{Y} \tau_{\mathrm{GL}_{p}}^{Y} \otimes \tau_{\mathrm{GL}_{q}}^{Y}
$$

where the sum is taken over Young diagrams $Y$ with at $\operatorname{most} \min (p, q)$ rows (see Thm 2.1.2 in [Ho2]). Recall that a joint highest weight vector of $\tau_{\mathrm{GL}_{p}}^{Y} \otimes \tau_{\mathrm{GL}_{q}}^{Y}$ is given as a product of determinants of $r \times r$ square blocks located in the upper left hand corner of $\mathfrak{p}$. If $Y$ has at most $m$ rows then the blocks needed are of size $r \leq m$ and the highest weight vector does not vanish on the matrix $P$. Hence it generates a representation of $\bar{K}_{\mathbb{C}}$ isomorphic to $\tau_{p}^{\lambda} \otimes \tau_{q}^{(\lambda, 0)}$ in $\operatorname{Sym}(\mathfrak{p})$ as well as in $\Gamma\left(\overline{\mathcal{O}}_{2^{p-1}}^{K}\right)$. The lemma is proved.

Let $J$ be the prime ideal in $\operatorname{Sym}(\mathfrak{p})$ corresponding to $\overline{\mathcal{O}}_{2^{p-1}}^{K}$. Since $I$ and $J$ have the same Krull dimension and $J$ is prime, in order to show that $J=I$, it suffices to show that $J \subseteq I$. We need the following lemma.

Lemma 6.3. Let $\tau=\tau_{p}^{\gamma} \otimes \tau_{q}^{\gamma^{\prime}}$ be a $K_{\mathbb{C}}$-type in $\operatorname{Sym}^{n}(\mathfrak{p})$ which does not lie in the ideal I. Then $\gamma^{\prime}=(\gamma, 0, \ldots, 0)$ and $\tau$ is generated by a joint highest weight vector of the representation $\tau_{\mathrm{GL}_{p}}^{\left(\gamma, \mathbf{0}_{p-m}\right)} \otimes \tau_{\mathrm{GL}_{q}}^{\left(\gamma, \mathbf{0}_{q-m}\right)}$ in $\operatorname{Sym}^{n}(\mathfrak{p})$ in $(16)$.

Proof. Note that any irreducible summand of $\tau \otimes \tau_{\min }$ is isomorphic to $\tau_{p}^{\gamma+\nu} \otimes \tau_{q}^{\gamma^{\prime}}$ for some weight $\nu$ of $\tau_{p}^{\frac{q-p}{2}} \mathbf{1}$. Since $\tau$ is not contained in $I, \tau \cdot \tau_{\min } \neq 0$ in $\operatorname{Gr}_{n}(V)$. By (13), $\gamma^{\prime}=(\lambda, \mathbf{0})$ for some $\lambda$ and $\sum_{i} \lambda_{i}=n$ and

$$
\gamma+\nu=\lambda+\frac{q-p}{2} \mathbf{1}_{\frac{p-1}{2}}
$$


By the theory of $\mathrm{SO}_{p} \times \mathrm{GL}_{q}$ harmonics on $\mathfrak{p}^{*}$ (for example see [Ho1]), the representation $\tau_{p}^{\gamma}$ first appears in degree

$$
\sum \gamma_{i}=\sum_{i} \lambda_{i}+\frac{q-p}{2} m-\sum_{i} \nu_{i}=n+\frac{q-p}{2} m-\sum_{i} \nu_{i} \geq n .
$$

The last equality holds if and only if $\nu$ is the highest weight vector of $\tau_{p}^{\frac{q-p}{2}} \mathbf{1}$ ie $\nu=\frac{q-p}{2} \mathbf{1}$. Since, by the assumption, $\tau_{p}^{\gamma}$ occurs in the degree $n$ we must have $\lambda=\gamma$. On the other hand, the first occurrence of $\tau_{p}^{\gamma}$ happens in $\operatorname{Sym}^{n}(\mathfrak{p})$ within the harmonics. It follows that $\tau$ occurs in the harmonics $\tau_{p}^{\gamma} \otimes \tau_{\mathrm{GL}_{q}}^{\left(\gamma, \mathbf{0}_{q-m}\right)}$. By the branching rule from $\mathrm{GL}_{q}$ to $\mathrm{SO}_{q}, \tau_{\mathrm{GL}_{q}}^{\left(\gamma, \mathbf{0}_{q-m}\right)}$ contains $\tau_{q}^{(\gamma, \mathbf{0})}$ with multiplicity one and it is generated by the highest weight vector. This joint highest weight vector also generates $\tau_{\mathrm{GL}_{p}}^{\left(\gamma, \mathbf{0}_{p-m}\right)} \otimes \tau_{\mathrm{GL}_{q}}^{\left(\gamma, \mathbf{0}_{q-m}\right)}$ in $\operatorname{Sym}^{n}(\mathfrak{p})$. This proves the lemma.

Since the highest vector of $\tau_{\mathrm{GL}_{p}}^{\left(\gamma, \mathbf{0}_{p-m}\right)} \otimes \tau_{\mathrm{GL}_{q}}^{\left(\gamma, \mathbf{0}_{q-m}\right)}$ does not vanish on $P$, we have shown that any $K$-type outside $I$ is also outside $J$. This shows that $J \subseteq I$, as desired. Theorem 6.1 is proved.

Case $p-1 \geq q$. In this case we only state the results. Consider the partition $\left(2^{q}, 1^{p-q}\right)$. There is only one real form of this orbit for $\mathrm{O}(p, q)$. Indeed, since the number of odd rows is $p-q$ and the signature is $p-q$ we have to enter + in all rows of length one. Let $\mathcal{O}_{2^{q}}$ be the corresponding nilpotent $\mathrm{O}(p, q)$-orbit. By Theorem 9.3.4 [CM], $\mathcal{O}_{2^{q}}$ is a union of two $\mathrm{SO}(p, q)^{0}$-orbits, denoted by $\mathcal{O}_{2^{q}}^{+}$and $\mathcal{O}_{2^{q}}^{-}$respectively. Let $\mathcal{O}_{2^{q}}^{K,+}$ and $\mathcal{O}_{2^{q}}^{K,-}$ be

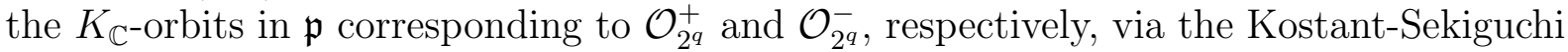
correspondence. If we identify $\mathfrak{p}$ with the space of complex $p \times q$ matrices then the two $K_{\mathbb{C}^{-} \text {-orbits }}$ are generated by elements

$$
P^{+}=E_{1,1}+\cdots+E_{m, m} \text { and } P^{-}=E_{1,1}+\cdots+E_{m-1, m-1}+E_{m, m+1} .
$$

Theorem 6.4. The associated variety of $V^{+}$and of $V_{o}^{+}$is the closure of the $K_{\mathbb{C}^{-} \text {-orbit }}$ $\mathcal{O}_{2^{q}}^{K,+}$. The associated variety of $V^{-}$and of $V_{o}^{-}$is the closure of the $K_{\mathbb{C}^{-} \text {orbit }} \mathcal{O}_{2^{q}}^{K,-}$.

One also could prove the above theorem for $V^{ \pm}$using the results of Knapp [Kn] and Trapa [T].

\section{LANGLANDS PARAMETERS}

In this section we will compute Langlands' parameters of $V, V^{ \pm}$and $V_{o}^{ \pm}$. In order to simplify some notation, let

$$
m=\min \left(\frac{p-1}{2}, \frac{q}{2}\right)
$$

Case $p \leq q-1$. Fix a minimal parabolic subgroup $P_{\min }=M_{\min } A_{\min } N_{\min }$. The root system of $G_{p, q}$ relative to $A_{\min }$ is $\mathrm{B}_{p}$. We shall realize this root system in a standard fashion, 
so that

$$
\alpha_{1}=\varepsilon_{1}-\varepsilon_{2}, \ldots, \alpha_{p-1}=\varepsilon_{p-1}-\varepsilon_{p} \text { and } \beta=\varepsilon_{p}
$$

are simple roots. Long root spaces are one-dimensional and for every long root $\alpha$ we have an embedding

$$
\varphi_{\alpha}: \widetilde{\mathrm{SL}}_{2}(\mathbb{R}) \rightarrow G_{p, q}
$$

where $\widetilde{\mathrm{SL}}_{2}(\mathbb{R})$ is the metaplectic cover $\mathrm{SL}_{2}(\mathbb{R})$. Let $Z_{\alpha}$ be the image, under $\varphi_{\alpha}$, of the center of $\widetilde{S L}_{2}(\mathbb{R})$. Note that $Z_{\alpha}$ is a cyclic group of order 4 . On the other hand, for every short root and $\beta$ in particular we have an embedding

$$
\varphi_{\beta}: \operatorname{Spin}(q-p+1,1) \rightarrow G_{p, q} .
$$

The existence of this embedding is a combination of two facts. First, the rank one Lie subalgebra of $\mathfrak{s o}(p, q)$ corresponding to $\beta$ is $\mathfrak{s o}(q-p+1,1)$ which gives a map from $\operatorname{Spin}(q-p+1,1)$ into $\operatorname{Spin}(p, q)$. Second, $\operatorname{Spin}(q-p+1,1)$ is topologically simply connected, so the map lifts to $G_{p, q}$. Let $Z_{\beta}$ be the image of the center of $\operatorname{Spin}(q-p+1,1)$. Then $Z_{\beta}$ is a cyclic group of order 2 .

Pseudo-spherical principal series. Here we define the principal series representations associated to the minimal parabolic subgroup. Note that the connected component of $M_{\min }$ is $\operatorname{Spin}(q-p)$, and

$$
M_{\text {min }}=\operatorname{Spin}(q-p) \times Z_{\beta} \times Z_{\alpha_{1}} \times_{\mu_{2}} \ldots \times_{\mu_{2}} Z_{\alpha_{p-1}}
$$

where $\mu_{2}=\{ \pm 1\}$ is the subgroup of $G_{p, q}$ such that $G_{p, q} / \mu_{2}$ is linear. Define

$$
M_{\min , \beta}=\operatorname{Spin}(q-p) \times Z_{\beta}
$$

Then $M_{\min } / M_{\min , \beta}$ is a Heisenberg group of order $2^{p}$. Let $S$ be the unique representation of this group such that the center $\mu_{2}$ acts via the unique non-trivial character. The dimension of $S$ is $2^{m}$.

Let $\chi \in \mathfrak{a}_{\min }^{*}$ be such that $\operatorname{Re}(\chi)$ is dominant. Then we have a pseudo-spherical, standard module (normalized induction)

$$
\operatorname{Ind}_{P_{\min }}[S \otimes \exp (-\chi)] \text {. }
$$

Generalized principal series. For every $k=1, \ldots, m$, the group $G_{p, q}$ has a parabolic subgroup $P=M A N$ - depending on $k$ - such that $A=\left(\mathbb{R}_{\text {long }}^{+}\right)^{m} \times \mathbb{R}_{\text {short }}^{+}$and the connected component of $M$ is

$$
M_{0}=\operatorname{Spin}(q-p) \times \widetilde{\mathrm{SL}}_{2}(\mathbb{R}) \times_{\mu_{2}} \ldots \times_{\mu_{2}} \widetilde{\mathrm{SL}}_{2}(\mathbb{R}) .
$$

where there are $m$ factors of $\widetilde{\mathrm{SL}}_{2}(\mathbb{R})$. More precisely,

(i) The factors of $A=\left(\mathbb{R}_{\text {long }}^{+}\right)^{m} \times \mathbb{R}_{\text {short }}^{+}$correspond to $m$ long roots $\varepsilon_{1}+\varepsilon_{2}, \varepsilon_{3}+$ $\varepsilon_{4}, \ldots, \varepsilon_{2 k-3}+\varepsilon_{2 k-2}, \varepsilon_{2 k}+\varepsilon_{2 k+1}, \ldots, \varepsilon_{p-1}+\varepsilon_{p}$ and the short root $\varepsilon_{2 k-1}$.

(ii) The factors $\widetilde{\mathrm{SL}}_{2}(\mathbb{R})$ in $(18)$ correspond to the following $m$ long roots: $\alpha_{1}, \alpha_{3}, \ldots$, $\alpha_{2 k-3}, \alpha_{2 k}, \alpha_{2 k+2}, \ldots, \alpha_{p-1}$. 
Define

$$
M_{\beta}=M_{0} \times Z_{\beta} .
$$

The quotient $M / M_{\beta}$ is a $\mathbb{Z} / 2 \mathbb{Z}$-vector space of rank $m$. It is spanned by $h_{\alpha}$, where $h_{\alpha}$ is an element of order 4 in $M_{\alpha}$ as $\alpha$ runs through the following $m$ long roots:

$$
\alpha_{2}, \alpha_{4}, \ldots, \alpha_{2 k-2}, \alpha_{2 k-1}, \alpha_{2 k+1}, \ldots, \alpha_{p-2}
$$

Assume now that $p<q-1$ so that $\frac{q-p}{2} \geq 3 / 2$. Let $D\left(\frac{q-p}{2}\right)^{+}$and $D\left(\frac{q-p}{2}\right)^{-}$be the holomorphic and anti-holomorphic discrete series representations of $\widetilde{\mathrm{SL}}_{2}(\mathbb{R})$ such that the lowest weight of $D\left(\frac{q-p}{2}\right)^{+}$is $\frac{q-p}{2}$ and the highest weight of $D\left(\frac{q-p}{2}\right)^{-}$is $-\frac{q-p}{2}$. The infinitesimal character of these two representation is $\frac{q-p-2}{2}$. Define a representation $W$ of $M$ by

$$
W=\operatorname{Ind}_{M_{\beta}}^{M}\left[\left(D\left(\frac{q-p}{2}\right)^{+}\right)^{\otimes m}\right]
$$

where $\operatorname{Spin}(q-p) \times Z_{\beta}$ acts trivially. We claim that $W$ is irreducible. Indeed, the restriction of $W$ to $M_{\beta}$ is a sum of $2^{m}$ terms of type

$$
D\left(\frac{q-p}{2}\right)^{\epsilon_{1}} \otimes \ldots \otimes D\left(\frac{q-p}{2}\right)^{\epsilon_{m}}
$$

where $\epsilon_{1}, \ldots, \epsilon_{m}= \pm$ (all possible choices). Since these summands are mutually nonisomorphic, irreducibility of $W$ follows at once from Mackey's criterion.

Define a standard module for $G_{p, q}$ by

$$
\operatorname{Ind}_{P}^{G_{p, q}}[W \otimes \exp (-\eta)]
$$

where

$$
\begin{aligned}
\eta & =\eta_{1}\left(\varepsilon_{1}+\varepsilon_{2}\right)+\ldots+\eta_{k} \varepsilon_{k}+\ldots+\eta_{m+1}\left(\varepsilon_{p-1}+\varepsilon_{p}\right) \\
& =\left(\eta_{1}, \eta_{1} \ldots, \eta_{k-1}, \eta_{k-1}, \eta_{k}, \eta_{k+1}, \eta_{k+1}, \ldots, \eta_{m+1}, \eta_{m+1}\right)
\end{aligned}
$$

and $\eta_{1}>\ldots>\eta_{m+1}>0$. This representation has a unique submodule with the minimal $K$-type $\tau_{p}^{\frac{q-p}{2}} \mathbf{1} \otimes \mathbb{C}$.

Theorem 7.1. We have the following:

(i) Suppose $q=p+1$. Then $V$ is the Langlands submodule of a normalized induced (pseudo-spherical) principal series representation (17) with

$$
\chi=\frac{1}{2}(p, p-1, p-2, \ldots, 1) .
$$

(ii) Suppose $q>p+1$. Then $V$ is the unique Langlands submodule of a normalized induced principal series representation in (19) where $k=1$ and

$$
\eta=\left(\eta_{1}, \ldots, \eta_{\frac{p+1}{2}}\right)=\left(\frac{p+q}{4}, \frac{p-1}{2}, \ldots, 2,1\right)+\frac{q-p-2}{4} \mathbf{1}_{\frac{p+1}{2}} .
$$

The submodule $V$ in each case is generated by the minimal $K$-type $\tau_{p}^{\frac{q-p}{2} \mathbf{1}} \otimes \mathbb{C}$ of the principal series representation. 
Proof. The first case, when $q=p+1$, is trivial since $V$ is a pseudospherical representation of the split group $G_{p, p+1}$. See also $[\mathrm{A}-\mathrm{V}]$ and [Wa2].

Lemma 7.2. Assume that $q>p+1$. Then $V$ is a submodule of $\operatorname{Ind}_{P}^{G_{p, q}}[W \otimes \exp (-\eta)]$ for some $k$.

Proof. This is a standard procedure so we only give a sketch. We use Proposition 4.1 in [Vo1]. There one constructs $\lambda$ in $i \mathfrak{t}_{0}^{*}$ from the minimal $K$-type. Its stabilizer in $\mathfrak{s o}_{p+q}(\mathbb{C})$ is a theta stable parabolic $\mathfrak{l}+\mathfrak{n}$ such that $\mathfrak{l}_{0}=\mathfrak{s u}_{m, m}+\mathfrak{s l}_{2}+\mathfrak{u}_{1}^{\frac{q-p-1}{2}}$. We may choose a split torus $\mathfrak{a}_{0}$ in $\mathfrak{l}_{0}$ such that $\mathfrak{a}_{0}$ is the Lie algebra of $A$ in $P=M A N$ in (18). The discrete series parameter on $M$ could be read off from $\lambda$.

Since the discrete series representation $D\left(\frac{q-p}{2}\right)^{+}$of $\widetilde{\mathrm{SL}}_{2}(\mathbb{R})$ embeds into the pseudospherical principal series representation of $\widetilde{S L}_{2}(\mathbb{R})$ with $\exp \left(\frac{q-p-2}{2}\right)$ on the "A" part of the minimal parabolic subgroup, it follows from induction in stages that

$$
\operatorname{Ind}_{P}[W \otimes \exp (-\eta)] \subseteq \operatorname{Ind}_{P_{\min }}[S \otimes \exp (-\chi)],
$$

where $\chi=\left(\chi_{1}, \ldots, \chi_{p}\right)=\eta+\xi$,

$$
\left\{\begin{array}{l}
\eta=\left(\eta_{1}, \eta_{1} \ldots, \eta_{k-1}, \eta_{k-1}, \eta_{k}, \eta_{k+1}, \eta_{k+1}, \ldots, \eta_{m+1}, \eta_{m+1}\right) \\
\xi=\frac{q-p-2}{4}(1,-1, \ldots, 1,-1,0,1,-1, \ldots, 1,-1)
\end{array}\right.
$$

and 0 is at the position $2 k-1$. Since the infinitesimal character of the pseudo-spherical principal series is $\operatorname{Ind}_{P_{\min }}[S \otimes \exp (-\chi)]$ is $\left(\chi, \rho_{q-p}\right)$ we see that $\left(\eta+\xi, \rho_{q-p}\right)$ is equal to $\mu_{p, q}$ up to a Weyl group element. Let $a=\frac{q-p-2}{4}$. If we remove $\rho_{q-p}$ from $\left(\eta+\xi, \rho_{q-p}\right)$ and $\mu_{p, q}$ then, up to a permutation of entries, we have

$$
\left(\eta_{1}+a, \eta_{1}-a, \ldots, \eta_{k-1}+a, \eta_{k-1}-a, \eta_{k}, \eta_{k+1}+a, \eta_{k+1}-a, \ldots, \eta_{m+1}+a, \eta_{m+1}-a\right)
$$

equals $\left(1,2, \ldots, \frac{p-1}{2}, \frac{q-p}{2}, \frac{q-p+2}{2}, \ldots, \frac{q-1}{2}\right)$.

If $k \neq 1$ then, by comparing the largest entries, $\eta_{1}+a=\frac{q-1}{2}$. This implies that the second entry $\eta_{1}-a=\frac{p+1}{2}$ which is not an entry of $\mu_{p, q}$. Hence $k=1$ and $\eta_{1}=\frac{q-1}{2}$. Since $q>p+1$, the next largest entry is $\frac{q-3}{2}$ and we conclude that $\eta_{2}+a=\frac{q-3}{2}$ and $\eta_{2}-a=\frac{p-1}{2}$. We can apply this argument repeatedly to conclude that $\eta$ has the desired form. Theorem 7.1 is proved.

In the above computation of the Langlands parameter, we only use the minimal $K$-type and infinitesimal character of $V$. This gives the following corollary.

Corollary 7.3. The module $V$ is the unique irreducible $(\mathfrak{s o}(p, q), K)$-module with the minimal $K$-type $\tau_{p}^{\frac{p-q}{2} \mathbf{1}} \otimes \mathbb{C}$ and infinitesimal character $\mu_{p, q}$. 
Next we consider $p-1 \geq q$ so $m=\frac{q}{2}$. Let $P_{\min }=M_{\min } A_{\min } N_{\min }$ be a minimal parabolic subgroup of $G_{p, q}$. The restricted root system for $A_{\min }$ is of type $\mathrm{B}_{q}$. We realize $\mathrm{B}_{q}$ so that the simple roots are

$$
\alpha_{1}=\varepsilon_{1}-\varepsilon_{2}, \ldots, \alpha_{q-1}=\varepsilon_{q-1}-\varepsilon_{q}, \text { and } \beta=\varepsilon_{q} .
$$

We assume that $N_{\min }$ is "spanned" by positive roots. We discuss the split $(p-1=q)$ and non-split $(p-1>q)$ cases separately.

Case $p-1>q$. Let $P=M A N$ be a parabolic subgroup of $G_{p, q}$ containing $P_{\min }$ such that the connected component $M_{0}$ of $M$ is

$$
M_{0}=\operatorname{Spin}(p-q) \times \widetilde{\mathrm{SL}}_{2}(\mathbb{R}) \times_{\mu_{2}} \ldots \times_{\mu_{2}} \widetilde{\mathrm{SL}}_{2}(\mathbb{R}) .
$$

Here there are $m$ copies of $\widetilde{\mathrm{SL}}_{2}(\mathbb{R})$ corresponding to the restricted roots $\varepsilon_{2 i-1}-\varepsilon_{2 i}$ for $i=1, \ldots, m$. Define

$$
M_{\beta}=M_{0} \times Z_{\beta} .
$$

Since $p-1>q$ we have $\frac{1}{2}(p-q) \geq \frac{3}{2}$. We recall that $D\left(\frac{p-q}{2}\right)^{+}$denotes the discrete series representation of $\widetilde{\mathrm{SL}_{2}}(\mathbb{R})$ with the lowest weight $\frac{p-q}{2}$ and $D\left(\frac{p-q}{2}\right)^{-}$is its dual module. Let $E=\left(\epsilon_{1}, \ldots, \epsilon_{m}\right)$ be an $m$-tuple of signs where $\epsilon_{i}= \pm$. We define

$$
W^{E}=\operatorname{Ind}_{M_{\beta}}^{M}\left[D\left(\frac{p-q}{2}\right)^{\epsilon_{1}} \otimes \ldots \otimes D\left(\frac{p-q}{2}\right)^{\epsilon_{m}}\right]
$$

where the subgroup $\operatorname{Spin}(p-q) \times Z_{\beta}$ acts trivially. The representations $W^{E}$ are irreducible by the Mackey irreducibility criterion. Indeed, the restriction of $W^{E}$ back to $M_{\beta}$ consists of summands

$$
D\left(\frac{p-q}{2}\right)^{\epsilon_{1}^{\prime}} \otimes \ldots \otimes D\left(\frac{p-q}{2}\right)^{\epsilon_{m}^{\prime}}
$$

for all possible combinations of signs $\left(\epsilon_{1}^{\prime}, \ldots, \epsilon_{m}^{\prime}\right)$ such that $\prod_{i=1}^{m} \epsilon_{i}^{\prime}=\prod_{i=1}^{m} \epsilon_{i}$. (Here we identify $\epsilon=+$ and - with $\epsilon=1$ and -1 respectively. There are $2^{m-1}$ such combinations which is precisely the index of $M_{\beta}$ in $M$.) Thus, not only are representations $W^{E}$ irreducible, but two such representations $W^{E}$ and $W^{E^{\prime}}$ are isomorphic if and only if the above product condition is satisfied. Thus, we have two isomorphism classes which we denoted by $W^{+}$and $W^{-}$, where the sign is $\prod_{i=1}^{m} \epsilon_{i}$.

Next, $A \cong\left(\mathbb{R}^{+}\right)^{m}$, with the coordinates given by the long roots $\varepsilon_{2 i-1}+\varepsilon_{2 i}, i=1, \ldots, m$. Let $\exp \eta$ denote a character of $A$ where $\eta=\sum_{i=1}^{m} \eta_{i}\left(\varepsilon_{2 i-1}+\varepsilon_{2 i}\right)$. If $\eta_{1}>\ldots>\eta_{m-1}>\left|\eta_{m}\right|$ then the induced representation

$$
\operatorname{Ind}_{P}^{G_{p, q}}\left(W^{ \pm} \otimes \exp (-\eta)\right)
$$

contains a unique irreducible submodule. Its minimal $K$-type is the minimal type of $V^{ \pm}$. 
Theorem 7.4. If $p>q+1$ then $V^{ \pm}$is the irreducible Langlands submodule of $\operatorname{Ind}_{P}^{G_{p, q}}\left[W^{ \pm} \otimes \exp (-\eta)\right]$ where

$$
\left(\eta_{1}, \ldots, \eta_{\frac{q}{2}}\right)=\left(\frac{q}{2}, \frac{q-2}{2}, \ldots, 1\right)+\frac{1}{4}(p-q-2) \mathbf{1}_{\frac{q}{2}} .
$$

Case $p-1=q$. Let $P=M A N$ be the parabolic subgroup in the standard position such that $M$ contains the following as a subgroup of finite index:

$$
M_{\beta}=Z_{\beta} \times \widetilde{\mathrm{SL}}_{2}(\mathbb{R}) \times_{\mu_{2}} \ldots \times_{\mu_{2}} \widetilde{\mathrm{SL}}_{2}(\mathbb{R}) .
$$

Here $\beta$ is the unique short simple root and, as before, $Z_{\beta}$ the image of of the center of $\operatorname{Spin}(2,1) \cong \mathrm{SL}_{2}(\mathbb{R})$ under $\varphi_{\beta}$. There are $m$ copies of $\widetilde{S L}_{2}(\mathbb{R})$, corresponding to the long simple roots $\alpha_{1}, \alpha_{3}, \ldots, \alpha_{2 m-1}$. Define an irreducible representation of $M$ - trivial on $Z_{\beta}$ - by

$$
W_{o}^{ \pm}=\operatorname{Ind}_{M_{\beta}}^{M}\left[D\left(\frac{3}{2}\right)^{\epsilon_{1}} \otimes \ldots \otimes D\left(\frac{3}{2}\right)^{\epsilon_{m}}\right]
$$

which, in an analogy with the case $p>q+1$, depends only on the sign of $\prod_{i=1}^{m} \epsilon_{i}$. Then the Langlands submodule of

$$
\operatorname{Ind}_{P}^{G_{p, q}}\left[W_{o}^{ \pm} \otimes \exp (-\eta)\right]
$$

contains the minimal $K$-type of $V_{o}^{ \pm}$.

The minimal parabolic $P_{\min }=M_{\min } A_{\min } N_{\min }$ of $M_{p, p-1}$ is the Borel subgroup. Let $\exp (\eta)$ be a dominant character of $A$ where $\eta=\sum_{i=1}^{q} \eta_{i} \varepsilon_{i}$. Let $S^{ \pm}$be the irreducible representation of $M_{\min }$ such that the Langlands submodule of the pseudo-spherical principal series representation

$$
\operatorname{Ind}_{P_{\min }}^{G_{p, p-1}}\left[S^{ \pm} \otimes \exp (-\eta)\right]
$$

contains the minimal $K$-type of $V^{ \pm}$.

Theorem 7.5. Here $p-1=q$ and $m=\frac{p-1}{2}=\frac{q}{2}$.

(i) $V^{ \pm}$is a pseudo-spherical submodule of $\operatorname{Ind}_{P_{\min }}^{G_{p, p-1}}\left[S^{ \pm} \otimes \exp (-\eta)\right]$ where

$$
\left(\eta_{1}, \ldots, \eta_{p-1}\right)=\frac{1}{2}(p-1, p-2, \ldots, 2,1) .
$$

(ii) $V_{o}^{ \pm}$is the irreducible Langlands submodule of $\operatorname{Ind}_{P}^{G_{p, p-1}}\left[W_{o}^{ \pm} \otimes \exp (-\eta)\right]$ where

$$
\left(\eta_{1}, \ldots, \eta_{m}\right)=(m, m-1, \ldots, 1)-\frac{1}{4} \mathbf{1}_{m}
$$

\section{An EXtension to DisconneCted GROUP}

The main purpose of this section is to extend $V$ to a $(\mathfrak{s o}(p, q), \operatorname{Spin}(p) \times \mathrm{O}(q))$-module. This is necessary to obtain a one to one correspondence when we next restrict $V$ to $\mathfrak{s o}(p, a) \times \mathrm{O}(b)$ where $a+b=q$, and $\mathrm{O}(b) \subseteq \mathrm{O}(q)$. 
Representations of $\mathrm{O}(n)$. We first describe a classification of irreducible representations of $\mathrm{O}(n)$. Let $\Lambda(\mathrm{O}(n))$ denote the subset of elements in $\mathbb{Z}^{n}$ such of the form

$$
\left(\lambda_{1}, \ldots, \lambda_{k}, \mathbf{0}_{n-k}\right) \text { or }\left(\lambda_{1}, \ldots, \lambda_{k}, \mathbf{1}_{n-2 k}, \mathbf{0}_{k}\right)
$$

where the $\lambda_{i}$ 's are positive integers, and $k \leq\left[\frac{n}{2}\right]$. Irreducible representations of $\mathrm{O}(n)$ are parameterized by $\Lambda(\mathrm{O}(n)$ ) as follows (see [GoW] or [Ho2]). Roughly speaking, for every $\lambda$ in $\Lambda(\mathrm{O}(n)), \tau_{\mathrm{O}(n)}^{\lambda}$ is the irreducible finite dimensional representation of $\mathrm{O}(n)$ generated by a highest weight vector of the finite dimensional representation of $\mathrm{GL}_{n}$ with the highest weight $\lambda$. In particular, note that

$$
\tau_{\mathrm{O}(n)}^{\left(\lambda_{1}, \ldots, \lambda_{k}, \mathbf{1}_{n-2 k}, \mathbf{0}_{k}\right)}=\operatorname{det}_{n} \otimes \tau_{\mathrm{O}(n)}^{\left(\lambda_{1}, \ldots, \lambda_{k}, \mathbf{0}_{n-k}\right)} .
$$

Elements of $\Lambda(\mathrm{O}(n))$ are called highest weights of $\mathrm{O}(n)$. Given a highest weight $\lambda$ in $\Lambda(\mathrm{O}(n))$, we define

$$
c(\lambda)=\left(\lambda_{1}, \ldots, \lambda_{k}, \mathbf{0}_{[n / 2]-k}\right) \in \Lambda(n) .
$$

The restriction of $\tau_{\mathrm{O}(n)}^{\lambda}$ to $\mathrm{SO}(n)$ is irreducible and isomorphic to $\tau_{n}^{c(\lambda)}$ unless $n=2 k$ and $\lambda_{k}>1$. In this case $\tau_{\mathrm{O}(n)}^{\lambda}$ is isomorphic to a direct sum $\tau_{n}^{c(\lambda)} \oplus \tau_{n}^{\sigma(c(\lambda))}$. In any case the infinitesimal character of $\tau_{\mathrm{O}(n)}^{\lambda}$ is $c(\lambda)+\rho_{n}$.

Next we discuss branching rule and tensor which are well known. Let $\lambda=\left(\lambda_{1}, \ldots, \lambda_{n}\right) \in$ $\Lambda(\mathrm{O}(n))$ and $\lambda^{\prime}=\left(\lambda_{1}^{\prime}, \ldots, \lambda_{n-1}^{\prime}\right) \in \Lambda(\mathrm{O}(n-1))$. We write $\lambda \succ_{\mathrm{O}} \lambda^{\prime}$ if

$$
\lambda_{1} \geq \lambda_{1}^{\prime} \geq \lambda_{2} \geq \ldots \geq \lambda_{n-1}^{\prime} \geq \lambda_{n} .
$$

The restriction of $\tau_{\mathrm{O}(n)}^{\lambda}$ to $\mathrm{O}(n-1)$ is given by

$$
\tau_{\mathrm{O}(n)}^{\lambda}=\oplus_{\lambda \succ \mathrm{O}} \lambda^{\prime} \tau_{\mathrm{O}(n-1)}^{\lambda^{\prime}} .
$$

This branching rule can be used to define another Gelfand-Zetlin basis of unit vectors of $\tau_{\mathrm{O}(n)}^{\lambda}$ by successive restrictions. Then each vector is represented by an array $N=\left(\lambda, \lambda^{1}, \ldots, \lambda^{n-1}\right)$ such that $\lambda^{i} \in \Lambda(\mathrm{O}(n-i))$ and $\lambda^{i-1} \succ_{\mathrm{O}} \lambda^{i}$. We will denote the corresponding basis vector by $\mathbf{v}_{N}^{\mathrm{O}}$. We warn that this basis is closely related but different from the Gelfand-Zetlin basis of $\mathfrak{s o}(n)$ introduced in Section 3. Let $N^{+s i}$ (respectively $N^{-s i}$ ) denote the array obtained from $N$ by adding (respectively subtracting) 1 from the $s$-th entry of $\lambda^{i}$.

Lemma 8.1. Let $I_{i+1, i}$ be the element in $\mathfrak{s o}(n)$ introduced in the beginning of Section 3. Then

$$
I_{i+1, i} \mathbf{v}_{N}^{\mathrm{O}}=\sum_{s=1}^{i} \alpha_{s} \mathbf{v}_{N^{+s, n-i}}^{\mathrm{O}}+\sum_{s=1}^{i} \beta_{s} \mathbf{v}_{N^{-s, n-i}}^{\mathrm{O}}
$$

where the number $\alpha_{s}$ (respectively $\beta_{s}$ ) is non-zero as long as the array $N^{+s, n-i}$ (respectively $\left.N^{-s, n-i}\right)$ satisfies the Gelfand-Zetlin pattern. 
Proof. We only give a sketch. First, note that it suffices to prove the statement in the case $i+1=n$. In this case the proof involves writing out the basis vectors $\mathbf{v}_{N}^{\mathrm{O}}$ in terms of the basis vectors $\mathbf{v}_{N}$ and then applying the formula for the action of $I_{n, n-1}$ on the basis $\mathbf{v}_{N}$ given on the page 364 in $[\mathrm{VK}]$.

Extension. We now extend $V$ to a $(\mathfrak{s o}(p, q), \operatorname{Spin}(p) \times \mathrm{O}(q))$-module. This extension will be very convenient for investigating dual pairs correspondences. There are two possible extensions and one differs from the other by the determinant character of $\mathrm{O}(p, q)$. Let $\varsigma$ be the diagonal matrix $\operatorname{diag}(1, \ldots, 1,-1)$ in $\mathrm{O}(q)$. We define an action of $\varsigma$ on the basis vector $\mathbf{v}_{M} \otimes \mathbf{v}_{N}$ by $(-1)^{n_{1}-n_{2}}$ where $n_{1}$ and $n_{2}$ are the sums of the entries of the top and second top rows of the Gelfand-Zetlin array $N$ respectively. With respect to this extension $V$ has $\operatorname{Spin}(p) \times \mathrm{O}(q)$-types

$$
\bigoplus_{\lambda \in \Lambda(p, 0)} \tau_{p}^{\lambda+\frac{p-q}{2} \mathbf{1}} \otimes \tau_{\mathrm{O}(q)}^{(\lambda, \mathbf{0})}
$$

where, we abbreviated, $\mathbf{1}=\mathbf{1}_{\frac{p-1}{2}}$ and $\mathbf{0}=\mathbf{0}_{q-\frac{p-1}{2}}$. Note that $V$ has a basis consisting of vectors $\mathbf{v}_{M} \otimes \mathbf{v}_{N}^{\mathrm{O}}$.

\section{Compact dual Pair CORRespondences}

In this section we restrict the $(\mathfrak{s o}(p, q)$, $\operatorname{Spin}(p) \times \mathrm{O}(q))$-module $V$ to $\mathfrak{s o}(p, a) \times \mathrm{O}(b)$ where $a+b=q$ and $\mathrm{O}(b)$ is included in $\mathrm{O}(q)$ in a standard way. Since $V$ is $\operatorname{Spin}(p)$-admissible, we have a direct sum

$$
V=\bigoplus_{\lambda^{\prime} \in \Lambda(\mathrm{O}(b))} \Theta\left(\lambda^{\prime}\right) \otimes \tau_{\mathrm{O}(b)}^{\lambda^{\prime}}
$$

where $\Theta\left(\lambda^{\prime}\right)$ are admissible and unitarizable Harish-Chandra modules of $G_{p, a}$. Every summand on the right hand side of (23) is spanned by basis vectors $\mathbf{v}_{M} \otimes \mathbf{v}_{N}^{\mathrm{O}}$ of $V$ where $\lambda^{q-b}=\lambda^{\prime}$ in $N$. We now state the main theorem of this section.

Theorem 9.1. Let $\lambda^{\prime} \in \Lambda(\mathrm{O}(b))$ and $\Theta\left(\lambda^{\prime}\right)$ defined in (23). If $\Theta\left(\lambda^{\prime}\right)$ is nonzero, then it is an irreducible unitarizable Harish-Chandra module of $G_{p, a}$. Moreover, if $\lambda^{\prime} \neq \gamma^{\prime}$ then $\Theta\left(\lambda^{\prime}\right)$ and $\Theta\left(\gamma^{\prime}\right)$ are not isomorphic.

Remark 9.2. Theorem A in Part II of $[\mathrm{KO}]$ proves a similar result for the ladder representation of $\mathrm{O}(p, q)$ where $p+q$ is even. Also compare with Theorem 3 in [Ko1] and, $[\mathrm{GW}]$.

Proof. We first describe the minimal $\operatorname{Spin}(p) \times \mathrm{SO}(a)$-type of $\Theta\left(\lambda^{\prime}\right)$. To this end, for any $n$-tuple $x=\left(x_{1}, \ldots, x_{n}\right)$ of real numbers define its height to be

$$
|x|=\sum_{i=1}^{n}\left|x_{i}\right| .
$$


If $\Theta\left(\lambda^{\prime}\right) \neq 0$ then $\tau_{\mathrm{O}(b)}^{\lambda^{\prime}}$ is contained in $\tau_{\mathrm{O}(q)}^{(\lambda, \mathbf{0})}$ for some $\lambda$ in $\Lambda(p, 0)$. It follows, from branching rules from $\mathrm{O}(q)$ to $\mathrm{O}(b)$, that the number of non-zero entries in $\lambda^{\prime}$ is less than or equal to

$$
u=\min \left(b, \frac{p-1}{2}\right) .
$$

In other words, we can write $\lambda^{\prime}=\left(\lambda_{1}^{\prime}, \ldots, \lambda_{u}^{\prime}, 0, \ldots, 0\right)$. Furthermore, the smallest height $\lambda$ in $\Lambda(p, 0)$ such that $\tau_{\mathrm{O}(q)}^{(\lambda, \mathbf{0})}$ contains $\tau_{\mathrm{O}(b)}^{\lambda^{\prime}}$ is

$$
\lambda=\left(\lambda_{1}^{\prime}, \ldots, \lambda_{u}^{\prime}, 0, \ldots, 0\right)
$$

and, in this case, $\tau_{\mathrm{O}(b)}^{\lambda^{\prime}}$ is contained in $\tau_{\mathrm{O}(q)}^{(\lambda, \mathbf{0})}$ with multiplicity one. It follows that $\mathrm{SO}(a)$ acts on this summand trivially. Summarizing, we have shown that the $\operatorname{Spin}(p) \times \mathrm{SO}(a)$ type

$$
\tau_{p}^{\lambda+\frac{q-p}{2} \mathbf{1}} \otimes \mathbb{C}
$$

appears with multiplicity one in $\Theta\left(\lambda^{\prime}\right)$ where $\lambda$ is given in terms of $\lambda^{\prime}$ as in (24). It is the minimal type of $\Theta\left(\lambda^{\prime}\right)$. Clearly, if $\lambda^{\prime} \neq \gamma^{\prime}$, then the minimal $\operatorname{Spin}(p) \times \operatorname{SO}(a)$-types of $\Theta\left(\lambda^{\prime}\right)$ and $\Theta\left(\gamma^{\prime}\right)$ are distinct.

It remains to show that $\Theta\left(\lambda^{\prime}\right)$ is irreducible. We will prove this by induction on $a$. When $a=1$, this is Proposition 4.1. By a see-saw pair argument, the restriction of $\Theta\left(\lambda^{\prime}\right)$ to $\mathfrak{s o}(p, a-1)$ decomposes as a direct sum

$$
\Theta\left(\lambda^{\prime}\right)=\oplus_{\lambda^{\prime \prime}} \Theta\left(\lambda^{\prime \prime}\right)
$$

where the sum is taken over all $\lambda^{\prime \prime} \in \Lambda(\mathrm{O}(b+1))$ such that $\lambda^{\prime \prime} \succ_{\mathrm{O}} \lambda^{\prime}$.

Suppose $\Theta\left(\lambda^{\prime}\right)$ is reducible. Then it decomposes completely since it is admissible and unitarizable. Let $\Pi$ be a proper submodule of $\Theta\left(\lambda^{\prime}\right)$ which does not contain the minimal $\operatorname{Spin}(p) \times \mathrm{SO}(a)$-type (25). By the induction assumption, the summands on the right hand side of (26) are irreducible and mutually non-isomorphic. Hence $\Pi$ is a direct sum of some summands $\Theta\left(\lambda^{\prime \prime}\right)$ in (26). Let $\Theta\left(\lambda^{\prime \prime}\right)$ be a summand of $\Pi$ such that the height of $\lambda^{\prime \prime}$ is minimal. Since $\Pi$ does not contain the minimal type (25),

$$
\left|\lambda^{\prime \prime}\right|>\left|\lambda^{\prime}\right|
$$

This shows that for some $s$ we have $\lambda^{\prime \prime}-\varepsilon_{s} \succ_{\mathrm{O}} \lambda^{\prime}$. Notice that the intersection

$$
\left(\Theta\left(\lambda^{\prime \prime}\right) \otimes \tau_{\mathrm{O}(b+1)}^{\lambda^{\prime \prime}}\right) \cap\left(\Theta\left(\lambda^{\prime}\right) \otimes \tau_{\mathrm{O}(b)}^{\lambda^{\prime}}\right)
$$

contains a vector of the form $\mathbf{v}_{M} \otimes \mathbf{v}_{N}^{\mathrm{O}}$ where the array $N$ contains $\lambda^{\prime \prime} \succ_{\mathrm{O}} \lambda^{\prime}$. By Lemma 8.1 $I_{p+a, p+a-1}$, acting on this vector, gives a non-zero summand involving $\mathbf{v}_{M} \otimes \mathbf{v}_{N^{\prime}}^{\mathrm{O}}$ where $N^{\prime}$ is obtained from $N$ by replacing $\lambda^{\prime \prime}$ by $\lambda^{\prime \prime}-\varepsilon_{s}$. In other words $\Pi$ contains $\Theta\left(\lambda^{\prime \prime}-\varepsilon_{s}\right)$. However, this contradicts the assumption that the height $\left|\lambda^{\prime \prime}\right|$ is minimal for $\Theta\left(\lambda^{\prime \prime}\right)$ contained in $\Pi$. Hence $\Theta\left(\lambda^{\prime}\right)$ is irreducible. The theorem is proved. 


\section{ACtion of Casimir operators}

Let $a$ and $b$ be a pair of non-negative integers such that $a+b=q$. Then $\mathfrak{s o}(p, a) \oplus \mathfrak{s o}(b)$ is a dual pair in $\mathfrak{s o}(p, q)$. In this section we shall compute a matching of the Casimir operators of the two Lie algebras acting on $V$.

Let $\Omega_{n}$ denote the Casimir operator of $\mathfrak{s o}(n)$. We remind the reader that $\Omega_{n}$ acts by the scalar $\|\lambda\|^{2}-\left\|\rho_{n}\right\|^{2}$ on a representation of $\mathfrak{s o}(n)$ with the infinitesimal character $\lambda$, and that the infinitesimal character of $\tau_{n}^{\lambda^{\prime}}$ is $\lambda^{\prime}+\rho_{n}$. Likewise, for $\lambda^{\prime} \in \Lambda(\mathrm{O}(n)), \tau_{\mathrm{O}(n)}^{\lambda^{\prime}}$ has infinitesimal character $c\left(\lambda^{\prime}\right)+\rho_{n}$ where $c\left(\lambda^{\prime}\right)$ was defined in (22).

Proposition 10.1. Let $\Omega_{p+a}$ and $\Omega_{b}$ be the Casimir elements of $\mathfrak{s o}(p, a)$ and $\mathfrak{s o}(b)$, respectively. Then

$$
\Omega_{p+a}-\Omega_{b}+\left(\frac{p-1}{2}\right)\left(\frac{q-1}{2}\right)\left(\frac{p+q}{2}-b\right)
$$

annihilates $V$.

Proof. In order to simplify notation we shall work with $\mathfrak{s o}_{p+q}(\mathbb{C})$ instead of $\mathfrak{s o}(p, q)$. (The Casimir operator is invariant of the choice of real form.) Recall that the algebra $\mathfrak{s o}_{p+q}(\mathbb{C})$ can be identified with the set of skew-symmetric matrices. The Casimir operator of $\mathfrak{s o}_{p+q}(\mathbb{C})$ is equal to

$$
\Omega_{p+q}=-\sum_{1 \leq i<j \leq p+q} I_{i j}^{2} .
$$

Clearly, in order to prove the lemma, it suffices to show that the operator annihilates every summand $\Theta\left(\lambda^{\prime}\right) \otimes \tau_{\mathrm{O}(b)}^{\lambda^{\prime}}$ in (23). Since the factors of any summand are irreducible representations, we know that is that $\Omega_{p+a}$ and $\Omega_{b}$ act as scalars on the summand. Thus, in order to evaluate $\Omega_{p+a}$ on $\Theta\left(\lambda^{\prime}\right)$, it suffices to do so on a carefully chosen vector. We define, as in the previous section,

$$
\lambda=\left(\lambda_{1}^{\prime}, \ldots, \lambda_{u}^{\prime}, 0, \ldots, 0\right) \in \Lambda(p, 0) .
$$

Then the restriction of $\tau_{\mathrm{O}(q)}^{(\lambda, 0)}$ to $\mathrm{O}(b)$ contains $\tau_{\mathrm{O}(b)}^{\lambda^{\prime}}$ with multiplicity one. It follows that we have a $\operatorname{Spin}(p) \times \mathrm{SO}(a) \times \mathrm{O}(b)$-type

$$
\delta=\tau_{p}^{\lambda+\frac{q-p}{2} \mathbf{1}} \otimes \mathbb{C} \otimes \tau_{\mathrm{O}(b)}^{\lambda^{\prime}}
$$

in $\Theta\left(\lambda^{\prime}\right) \otimes \tau_{\mathrm{O}(b)}^{\lambda^{\prime}}$. Since $\tau_{\mathrm{O}(b)}^{\lambda^{\prime}}$ is contained in $\tau_{\mathrm{O}(q-1)}^{\left(\lambda^{\prime}, \mathbf{0}_{q-b-1}\right)} \subseteq \tau_{\mathrm{O}(q)}^{\left(\lambda^{\prime}, \mathbf{0}_{q-b}\right)}$ it follows from Proposition 4.1 that $\delta$ is contained in

$$
\operatorname{Ind}_{p, 1}\left(\lambda+\frac{q-p}{2} \mathbf{1}, 0\right) \otimes \tau_{\mathrm{O}(q-1)}^{\left(\lambda^{\prime}, \mathbf{0}\right)} .
$$

Since the infinitesimal character of a principal series $\operatorname{Ind}_{p, 1}(\mu, 0)$ is $\left(\mu+\rho_{p-1}, 0\right)$, a simple calculation shows that

$$
\Omega_{p+1}-\Omega_{p}=-\sum_{i=1}^{p} I_{p+1, i}^{2}=-\left(\frac{p-1}{2}\right)\left(\frac{q-1}{2}\right)-\left|\lambda^{\prime}\right|
$$


on $\delta$. Let $j$ be an integer such that $p+1<j \leq p+a$, and $w$ a Weyl group element which permutes $\varepsilon_{p+1}$ and $\varepsilon_{j}$. We can pick a representative of $w$ in $\operatorname{SO}(a)$. Since $w\left(-\sum_{i=1}^{p} I_{p+1, i}^{2}\right)=-\sum_{i=1}^{p} I_{j i}^{2}$ and $\mathrm{SO}(a)$ acts trivially on $\delta$, the sums $-\sum_{i=1}^{p} I_{j i}^{2}$ for $p+1<j \leq p+a$ act by the same scalar on $\delta$. Summing over all $j$, we get that

$$
-\sum_{j=p+1}^{p+a} \sum_{i=1}^{p} I_{j i}^{2}=-a\left(\frac{p-1}{2}\right)\left(\frac{q-1}{2}\right)-a\left|\lambda^{\prime}\right|
$$

on $\delta$. Since

$$
\Omega_{p+a}=\Omega_{p}+\Omega_{a}-\sum_{j=p+1}^{p+a} \sum_{i=1}^{p} I_{j i}^{2}
$$

and the values of $\Omega_{p}, \Omega_{a}$ and $\Omega_{b}$ on $\delta$ are easily calculated, lemma is reduced to a straightforward check.

Proposition 10.2. Let $\lambda^{\prime}$ in $\Lambda(b, 0)$ such that $\Theta\left(\lambda^{\prime}\right) \neq 0$. Recall that the infinitesimal character of $\tau_{\mathrm{O}(b)}^{\lambda^{\prime}}$ is

$$
\lambda=c\left(\lambda^{\prime}\right)+\rho_{b}=\left(\lambda_{1}, \ldots, \lambda_{\left[\frac{b}{2}\right]}\right) .
$$

Let $r=\min \left(\left[\frac{b}{2}\right], \frac{p-1}{2}\right)$. Then there exists numbers $\nu_{r+1}, \ldots, \nu_{\left[\frac{p+a}{2}\right]}$ independent of $\lambda^{\prime}$ such that the infinitesimal character of $\Theta\left(\lambda^{\prime}\right)$ is $\left(\lambda_{1}, \ldots, \lambda_{r}, \nu_{r+1}, \ldots, \pm \nu_{\left[\frac{p+a}{2}\right]}\right)$. In particular, $V$ establishes a correspondence

$$
\left(\lambda_{1}, \ldots, \lambda_{r}, \nu_{r+1}, \ldots, \pm \nu_{\left[\frac{p+a}{2}\right]}\right) \longleftrightarrow\left(\lambda_{1}, \ldots, \lambda_{r}, \rho_{b-2 r}\right)
$$

for the dual pair $\mathfrak{s o}(p, a) \oplus \mathfrak{s o}(b)$.

Proof. Let $\nu=\left(\nu_{1}, \ldots, \nu_{\left[\frac{p+a}{2}\right]}\right)$ be the infinitesimal character of $\Theta\left(\lambda^{\prime}\right)$. Assume that $\lambda^{\prime \prime}=$ $\lambda^{\prime} \pm \varepsilon_{i}$ is a highest weight for some $i \leq \min \left(b, \frac{p-1}{2}\right)$. Then Lemma 8.1 shows that the action of $\mathfrak{s o}(p, q)$ on $\Theta\left(\lambda^{\prime}\right) \otimes \tau_{\mathrm{O}(b)}^{\lambda^{\prime}}$ followed by the projection on $\Theta\left(\lambda^{\prime \prime}\right) \otimes \tau_{\mathrm{O}(b)}^{\lambda^{\prime \prime}}$ is non-zero. Since

$$
\mathfrak{s o}(p, q)=\mathfrak{s o}(p, a) \oplus \mathfrak{s o}(b) \oplus \mathfrak{p}_{0}
$$

where $\mathfrak{p}_{0} \otimes \mathbb{C}=\mathbb{C}^{p+a} \otimes \mathbb{C}^{b}$, it follows that $\Theta\left(\lambda^{\prime \prime}\right)$ is a subquotient of $\mathbb{C}^{p+a} \otimes \Theta\left(\lambda^{\prime}\right)$. This shows that the infinitesimal character of $\Theta\left(\lambda^{\prime \prime}\right)$ is $\nu \pm \varepsilon_{j}$ for some $j$ or it is equal to $\nu$. The last possibility might happen only for $p+a$ odd, ie $b$ is even.

Before we state the next lemma we note that the infinitesimal character of $\tau_{\mathrm{O}(b)}^{\lambda^{\prime \prime}}$ is

$$
c\left(\lambda^{\prime \prime}\right)+\rho_{b}=\left\{\begin{array}{l}
\lambda-\varepsilon_{i} \text { if } \lambda^{\prime \prime}=\lambda^{\prime}-\varepsilon_{i} \text { or } \lambda^{\prime \prime}=\lambda^{\prime}+\varepsilon_{b-i+1} \text { for some } i<\frac{b+1}{2} \\
\lambda+\varepsilon_{i} \text { if } \lambda^{\prime \prime}=\lambda^{\prime}+\varepsilon_{i} \text { or } \lambda^{\prime \prime}=\lambda^{\prime}-\varepsilon_{b-i+1} \text { for some } i<\frac{b+1}{2} \\
\lambda \text { if } \lambda^{\prime \prime}=\lambda^{\prime} \pm \varepsilon_{\frac{b+1}{2}} .
\end{array}\right.
$$

The last case occurs only for $b$ odd.

Lemma 10.3. Let $\lambda^{\prime}$ and $\lambda^{\prime \prime}$ as above. Assume that for some $\lambda^{\prime}$ the infinitesimal character of $\Theta\left(\lambda^{\prime}\right)$ is given by $\nu=\left(\lambda_{1}, \ldots, \lambda_{r}, \nu_{r+1}, \ldots, \nu_{\left[\frac{p+a}{2}\right]}\right)$ for some $\nu_{i}$. 
(i) Suppose that the infinitesimal character of $\tau_{\mathrm{O}(b)}^{\lambda^{\prime \prime}}$ is $\lambda+\varepsilon_{i}$ for some $i \leq r$. Then the infinitesimal character of $\Theta\left(\lambda^{\prime \prime}\right)$ is $\nu+\varepsilon_{i}$.

(ii) Suppose that the infinitesimal character of $\tau_{\mathrm{O}(b)}^{\lambda^{\prime \prime}}$ is $\lambda-\varepsilon_{i}$ for some $i \leq r$. Then the infinitesimal character of $\Theta\left(\lambda^{\prime \prime}\right)$ is $\nu-\varepsilon_{i}$.

(iii) Suppose that the infinitesimal character of $\tau_{\mathrm{O}(b)}^{\lambda^{\prime \prime}}$ is $\lambda$. This happens only if $b$ is odd and $\lambda^{\prime \prime}=\lambda^{\prime} \pm \varepsilon_{\frac{b+1}{2}}$. Then the infinitesimal character of $\Theta\left(\lambda^{\prime \prime}\right)$ is $\sigma(\nu)$.

Proof. First, we claim that the infinitesimal character of $\Theta\left(\lambda^{\prime \prime}\right)$ is not equal to $\nu$ if $b$ is even. If the infinitesimal character of $\Theta\left(\lambda^{\prime}\right)$ is $\nu$ then the matching of Casimir operators in Proposition 10.1 implies that $\left\|\lambda \pm \varepsilon_{i}\right\|^{2}=\|\lambda\|^{2}$ and $\lambda_{i}=\mp 1 / 2$. However, $\lambda_{i}$ is an integer since $b$ is even. This is a contradiction and it proves our claim.

Let us prove (i). The infinitesimal character of $\Theta\left(\lambda^{\prime \prime}\right)$ is $\nu \pm \varepsilon_{j}$ for some $j$. Assume first that it is $\nu+\varepsilon_{j}$. Then Proposition 10.1 implies that

$$
\left\|\nu+\varepsilon_{j}\right\|^{2}-\|\nu\|^{2}=\left\|\lambda+\varepsilon_{i}\right\|^{2}-\|\lambda\|^{2}
$$

that is, $\nu_{j}=\lambda_{i}$. Permutation of $i$-th and $j$-th places - as an element of the absolute Weyl group of $\mathfrak{s o}(p, a)$ - replaces $\nu+\varepsilon_{j}$ by $\nu+\varepsilon_{i}$, as desired. Similarly, if the infinitesimal character is $\nu-\varepsilon_{i}$, then $\nu_{j}=-\lambda_{i}$. If $i \neq j$ then we can replace $\nu-\varepsilon_{j}$ by $\nu+\varepsilon_{i}$ by permuting the two places and changing the signs of both of them. If $i=j$ then $\nu_{i}=-\lambda_{i}$ implies that $\lambda_{i}=0$. It follows that $b$ is even and $p+a$ odd. Hence the absolute Weyl group of $\mathfrak{s o}(p, a)$ is a $\mathrm{B}$ type and $\nu+\epsilon_{i}$ can be replaced by $\nu-\epsilon_{i}$. The case (ii) is proved analogously. For the last case, the infinitesimal character of $\Theta\left(\lambda^{\prime \prime}\right)$ is $\nu \pm \varepsilon_{j}$ for some $j$. Then Proposition 10.1 implies that $\left\|\nu \pm \varepsilon_{j}\right\|^{2}=\|\nu\|^{2}$, that is, $\nu_{j}=\mp \frac{1}{2}$. It follows that $\nu \pm \varepsilon_{j}$ is Weyl group equivalent to $\sigma(\nu)$. The lemma is proved.

It remains to show that the infinitesimal character of $\Theta\left(\lambda^{\prime}\right)$ is of the desired form for one $\lambda^{\prime}$. Pick $\lambda^{\prime}$ so that $\lambda_{1}^{\prime}>\ldots>\lambda_{r}^{\prime}$. Then $\lambda^{\prime}+\varepsilon_{i}$ is a highest weight for all $i=1, \ldots, r$. Since the infinitesimal character of $\Theta\left(\lambda^{\prime}+\varepsilon_{i}\right)$ is equal to $\nu \pm \varepsilon_{j}$ for some $j$ it follows, from Proposition 10.1 that $\lambda_{i}= \pm \nu_{j}$. This shows that every $\lambda_{i}$ is up to a sign equal to an entry of $\nu$. Since the absolute rank of $\mathfrak{s o}(p, a)$ is bigger then $r$, the Weyl group can in any case rearrange the entries of $\nu$ so that it begins with $\lambda_{1}, \ldots, \lambda_{r}$.

Remark 10.4. The correspondence is independent of the real form of the complex dual pair $\mathfrak{s o}_{p+a}(\mathbb{C}) \oplus \mathfrak{s o}_{b}(\mathbb{C})$ in $\mathfrak{s o}_{p+q}(\mathbb{C})$.

Based on this observation, we can now give a proof of the first correspondence of infinitesimal characters in Theorem 1.2, that is, when $p \leq b \leq q$. Indeed, the above mentioned correspondence of infinitesimal characters is also equal to that of the dual pair $\mathfrak{s o}(p, b-p) \oplus \mathfrak{s o}(p+a)$. This uniquely determines the $\nu_{i}$ 's in Proposition 10.2 and proves the first correspondence in Theorem 1.2. 


\section{Correspondence of infinitesimal CharaCters}

In order to determine the correspondence of infinitesimal characters for the dual pair $\mathfrak{s o}_{p+a}(\mathbb{C}) \oplus \mathfrak{s o}_{b}(\mathbb{C})$ (Theorem 1.2) it remains to determine the $\nu_{i}$ 's in Proposition 10.2 if $b<p$. Recall that for every $p \leq q-1$ we have an embedding

$$
V \subseteq \operatorname{Ind}_{P_{\min }}[S \otimes \exp (-\chi)]
$$

where $\chi$ is given by Theorem 7.1 if $p=q-1$ and is constructed by means of $\eta$ as explained in $(21)$, otherwise. Next, for every $r=1, \ldots, p$ consider

$$
G_{r, r} \times_{\mu_{2}} G_{p-r, q-r} \subseteq G_{p, q}
$$

where the simple roots of $G_{r, r}$ are $\alpha_{1}, \alpha_{2}, \ldots, \alpha_{r-1}, \varepsilon_{r-1}+\varepsilon_{r}$.

Let $P^{\prime}=M^{\prime} A^{\prime} N^{\prime}$ and $P^{\prime \prime}=M^{\prime \prime} A^{\prime \prime} N^{\prime \prime}$ be the minimal parabolic subgroups of $G_{r, r}$ and $G_{p-r, q-r}$, respectively, in standard position with respect to our choices of simple roots. Let

$$
\begin{aligned}
\chi^{\prime} & =-\left(\chi_{1}, \ldots, \chi_{r}\right)+\left(\frac{p+q}{2}-r\right) \mathbf{1}_{r}, \\
\chi^{\prime \prime} & =-\left(\chi_{r+1}, \ldots, \chi_{p}\right) .
\end{aligned}
$$

Lemma 11.1. For every $r=1, \ldots, p$ there exists a nonzero homomorphism of $G_{r, r} \times$ $G_{p-r, q-r}$-modules

$$
V \rightarrow \operatorname{Ind}_{P^{\prime}}^{G_{r, r}}\left[S^{\prime} \otimes \chi^{\prime}\right] \otimes \operatorname{Ind}_{P^{\prime \prime}}^{G_{p-r, q-r}}\left[S^{\prime \prime} \otimes \chi^{\prime \prime}\right]
$$

for some $M^{\prime} \times M^{\prime \prime}$-summand $S^{\prime} \otimes S^{\prime \prime}$ of $S$. In particular - for this $\chi^{\prime}$ and $\chi^{\prime \prime}$ - we have a correspondence of infinitesimal characters

$$
\chi^{\prime} \longleftrightarrow\left(\chi^{\prime \prime}, \rho_{q-p}\right)
$$

Proof. Since $V$ is a submodule of $\operatorname{Ind}_{P_{\min }}[S \otimes(-\exp \chi)]$, the Frobenius reciprocity implies that there exists a non-trivial homomorphism of $P_{\min }$-modules $V \rightarrow S \otimes \exp (\rho-\chi)$. Restricting to $P^{\prime} \times P^{\prime \prime}$ and using the Frobenius reciprocity again, proves the lemma.

As we shall see in a moment, the correspondence (27) gives $\nu_{i}^{\prime}$ 's if $q-p \leq b<q$. In order to deal with $b<q-p$ we need one more statement. If $b<q-p$ then $a>p$. For such $a$ let $P_{a}=M_{a} A_{a} N_{a}$ be the minimal parabolic subgroup of $G_{p, a} \subseteq G_{p, q}$. In particular, we have the following obvious lemma:

Lemma 11.2. Assume that $a+b=q$ and $a>p$. There exists a non-zero homomorphism of $G_{p, a} \times \operatorname{Spin}(b)$-modules

$$
V \rightarrow \operatorname{Ind}_{P_{a}}^{G_{p, a}}[S \otimes \chi] \otimes \mathbb{C} .
$$

In particular we have a correspondence of two the infinitesimal characters

$$
\left(-\chi+\frac{b}{2} \mathbf{1}_{p}, \rho_{a-p}\right) \longleftrightarrow \rho_{b} .
$$


Proof of Theorem 1.2. The first correspondence in the theorem was established in Remark 10.4. It remains to deal with $b<p$.

Case 1: $q=p+1$. In this case $\chi=\frac{1}{2}(p, p-1, \ldots, 1)$ and, up to a Weyl group action, (27) becomes

$$
\left(\frac{p-r}{2}, \frac{p-r-1}{2}, \ldots, \frac{p-2 r+1}{2}\right) \longleftrightarrow\left(\frac{p-r}{2}, \frac{p-r-1}{2}, \ldots, \frac{1}{2}\right) .
$$

If we cancel out the same numbers from both sides of the above correspondences until one side is empty, then the remaining numbers are $\nu_{i}$ 's. If $b$ is even, we use $r=b / 2$. Then the remaining terms are on the left side. They are

$$
\left(\frac{p-2 r}{2}, \frac{p-2 r-1}{2}, \ldots, \frac{1}{2}\right)=\left(\frac{p-b}{2}, \frac{p-b-1}{2} \ldots, \frac{1}{2}\right)
$$

and this is $\mu_{p-b, p-b+1}$, as desired. If $b$ is odd, then we use $r=(a+p) / 2$, and the remaining terms are on the right side. They are

$$
\left(0, \frac{-1}{2}, \ldots \frac{p-2 r+1}{2}\right)=\left(0,-\frac{1}{2}, \ldots,-\frac{p-b}{2}\right)
$$

and this is $\delta_{p-b+1, p-b+1}$, up to a Weyl group action. This proves the third correspondence. Case 2: $q>p+1$. We set $e=0$ if $r$ is even and $e=\frac{1}{2}$ if $r$ is odd. Let $m=\frac{p-1}{2}$ and

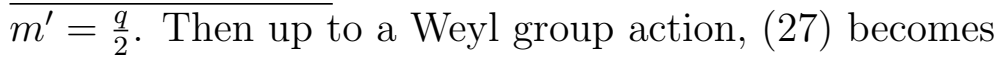

$$
\begin{aligned}
& \left(m-r+1, m-r+2, \ldots, m-\frac{r}{2}+e ; m^{\prime}-r+\frac{1}{2}, m^{\prime}-r+\frac{3}{2}, \ldots, m^{\prime}-\frac{r+1}{2}-e\right) \\
& \longleftrightarrow\left(1,2, \ldots, m-\frac{r}{2}+e ; \frac{1}{2}, \frac{3}{2}, \ldots, m^{\prime}-\frac{r+1}{2}-e\right) .
\end{aligned}
$$

Likewise, we remove the same set of numbers from both sides of the above correspondences until one side is empty. Then the numbers that are left behind would be the $\nu_{i}$ 's. Set $b=2 r$ and assume that $b<p$. Then $r<m, r<m^{\prime}$ and $\left(\nu_{i}\right)=\mu_{p-b, q-b}$. This gives the second correspondence of Theorem 1.2. If we set $p+a=2 r$ and assume that $b=p+q-2 r<p$ then, since $r \leq p$, we must also have that $b \geq q-p$. In this case we get $\left(\nu_{i}\right)=\delta_{p-b+1, q-b}$. This gives the third correspondence of Theorem 1.2 for the case $p>b \geq q-p$.

Finally if $b$ is odd, $b \leq q-p$ and $b<p$, then we refer to Lemma 11.2. Then up to a Weyl group action, (28) becomes

$$
\left(\rho_{b}, \rho_{p-b+1}, \rho_{q-b+1}\right) \longleftrightarrow \rho_{b}
$$

This gives $\left(\nu_{i}\right)=\delta_{p-b, q-b+1}$ and proves the third correspondence. With this, we complete the proof of Case 2 and also the proof of Theorem 1.2.

Since the annihilator of $V^{ \pm}$in $\mathfrak{s o}(p, q)$ is the same as the annihilator of $V$ in $\mathfrak{s o}(q+1, p-1)$ Theorem 1.1 also gives a matching of infinitesimal characters for $V^{ \pm}$except when $q=p-1$. In this case we have the following: 
Theorem 11.3. Assume $q=p-1$. Assume that $a$ and $b$ are positive integers such that $a+b=p-1$. Then $V^{+}, V^{-}, V_{o}^{+}$and $V_{o}^{-}$establish the following correspondence of infinitesimal characters for the dual pair $\mathfrak{s o}(p, a) \times \mathfrak{s o}(b)$ :

$$
\left\{\begin{array}{l}
\left(\lambda_{1}, \ldots, \lambda_{\frac{b}{2}}, \mu_{p-b, p-b-1}\right) \longleftrightarrow\left(\lambda_{1}, \ldots, \lambda_{\frac{b}{2}}\right) \text { if } b \text { is even } \\
\left(\lambda_{1}, \ldots, \lambda_{\frac{b-1}{2}}, \delta_{p-b, p-b}\right) \longleftrightarrow\left(\lambda_{1}, \ldots, \lambda_{\frac{b-1}{2}}\right) \text { if } b \text { is odd }
\end{array}\right.
$$

where we recall $\delta_{p-b, p-b}=\left(\rho_{p-b}, \rho_{p-b+1}\right)$ or $\left(\frac{p-b-1}{2}, \frac{p-b-2}{2}, \ldots, \frac{1}{2}, 0\right)$, up to a Weyl group action.

\section{REFERENCES}

[A-V] J. Adams, D. Barbasch, P. Trapa, A. Paul and D. Vogan, Shimura correspondences for split real groups. To appear in the Journal of AMS.

[Bo] N. Bourbaki, Lie groups and Lie algebras. Chapters 4-6. Translated from the 1968 French original by Andrew Pressley. Elements of Mathematics (Berlin). Springer-Verlag, Berlin, 2002. xii+300 pp.

[BFG1] Daniel Bump, S. Friedberg and D. Ginzburg, Small representations for odd orthogonal groups. Internat. Math. Res. Notices, 25 (2003) 1363-1393.

[BFG2] Daniel Bump, S. Friedberg and D. Ginzburg, Lifting automorphic representations on the double covers of odd orthogonal groups. Duke Math. J. 131 (2006) 363-396.

[BKo] R. Brylinski and B. Kostant, Minimal representations, geometric quantization, and unitarity. Proceeding of the National Academy of Science, USA, Vol 91, No 13 (1994), 6026-6029.

[CM] D. H. Collingwood and W. M. McGovern, Nilpotent orbits in semisimple Lie algebras. Van Nostrand Reinhold, NY, 1993.

[GoW] Roe Goodman and Nolan R. Wallach, Representations and Invariants of the Classical Groups Cambridge U. Press, 1998; third corrected printing, 2003.

[GW] B. Gross and Nolan R. Wallach, Restrictions of small representations to symmetric subgroups. Proc. Sympo. Pure Math 68 (2000), 255-272, AMS.

[Hi] T. Hirai, On infinitesimal operators of irreducible representations of the Lorentz group of $n$-th order. Proc. Japan Acad. 38 (1962) 83-87; On irreducible representations of the Lorentz group of $n$-th order. Proc. Japan Acad. 38 (1962) 258-262.

[Ho1] Roger Howe, Transcending classical invariant theory. J. Amer. Math. Soc. 2 (1989), no. 3, 535-552.

[Ho2] Roger Howe, Perspectives on invariant theory: Schur duality, multiplicity-free actions and beyond. The Schur lectures (1992) (Tel Aviv), 1-182, Israel Math. Conf. Proc., 8, Bar-Ilan Univ., Ramat Gan, 1995.

[HL] Jing-Song Huang and Jian-Shu Li, Unipotent representations attached to spherical nilpotent orbits. Amer. J. Math. 121 (1999), 497-517.

$[\mathrm{KG}]$ A. U. Klimyk and A. M. Gavrilik, The representations of the groups $\mathrm{U}(n, 1)$ and $\mathrm{SO}_{0}(n, 1)$, preprint ITP-76-39E, Instituent for Theoretical Physics Kiev, USSR,1976.

[Kn] Anthony Knapp, Nilpotent orbits and some unitary representations of indefinite orthogonal groups. Journal of Functional Analysis 209 (2004) 36-100.

[Ko1] T. Kobayashi, The Restriction of $A_{\mathfrak{q}}(\lambda)$ to reductive subgroups, Proc. Acad. Japan 69 (1993), 262-267.

[Ko2] T. Kobayashi, Discretely decomposability of the restriction of $A_{\mathfrak{q}}(\lambda)$ with respect to reductive subgroups and its applications Part I Invent. Math. 131 (1994), 229-256; Part II Ann. Math. 147 (1998), 709-729; Part III Invent. Math 131 (1998), 229-256. 
[KO] T. Kobayashi and B. Orsted, Analysis of the minimal representation of $\mathrm{O}(p, q)$ I, II, III. Adv. Math. 180, No 2 (2003) 486-595.

[Sa] H. Sabourin, Une représentation unipotent associee à l'orbit minimale: le cas de $\mathrm{SO}(4,3)$. Journal of Functional Analysis 137 (1996), 394-465.

[SV] Wilfried Schmid, and Kari Vilonen, Characteristic cycles and wave front cycles of representations of reductive Lie groups. Ann. of Math. (2) 151 (2000), no. 3, 1071-1118.

[To] P. Torasso, Méthod des orbits de Kirillov-Duflo et représentations minimales des groupes simples sur un corps local de caracteristique nulle. Duke Math. J 90 (1997) 261-377.

[T] Peter E. Trapa, Some small unipotent representations of indefinite orthogonal groups. Journal of Functional Analysis 213 (2004) 290-320.

[VK] N. Ja. Vilenkin and A. U. Klimyk, Representations of Lie groups and special functions, Vol 3: Classical and Quantum groups and special functions, Kluwer Academic Publishers, (1992).

[Vo1] David Vogan, The algebraic structure of the representation of semisimple Lie group I. Ann. of Math 100 (1979), 1-60.

[Vo2] David Vogan, Associated varieties and unipotent representations. Harmonic analysis on reductive groups (Brunswick, ME, 1989), 315-388, Progr. Math., 101, Birkhuser Boston, Boston, MA, 1991.

[Wa1] Nolan R. Wallach Real reductive groups I. Academic press, (1988).

[Wa2] Nolan R. Wallach, Real reductive groups II. Academic press, (1992).

[Zhe] D. P. Zhelobenko, Compact Lie Groups and Their Representations. Translations of Mathematical Monographs, Vol. 40, Amer. Math. Soc., Providence, RI, 1973.

[ZH] C.-B. Zhu and J.-S. Huang, On certain small representations of indefinite orthogonal groups. Representation theory 1, (1997), 190-206.

Hung Yean Loke, Department of Mathematics, National University of Singapore, 2 SCIENCE Drive 2, Singapore 117543

E-mail address: matlhy@nus.edu.sg

Gordan Savin, Department of Mathematics, University of Utah, Salt Lake City, Ut 84112

E-mail address: savin@math.utah.edu 